\title{
Network neuroscience and the connectomics revolution
}

\author{
Richard F. Betzel ${ }^{1-4 *}$ \\ ${ }^{1}$ Department of Psychological and Brain Sciences, \\ ${ }^{2}$ Cognitive Science Program, ${ }^{3}$ Program in Neuroscience, \\ ${ }^{4}$ Network Science Institute, Indiana University, Bloomington, IN 47405
}

(Dated: October 6, 2020)

\begin{abstract}
Connectomics and network neuroscience offer quantitative scientific frameworks for modeling and analyzing networks of structurally and functionally interacting neurons, neuronal populations, and macroscopic brain areas. This shift in perspective and emphasis on distributed brain function has provided fundamental insight into the role played by the brain's network architecture in cognition, disease, development, and aging. In this chapter, we review the core concepts of human connectomics at the macroscale. From the construction of networks using functional and diffusion MRI data, to their subsequent analysis using methods from network neuroscience, this review highlights key findings, commonly-used methodologies, and discusses several emerging frontiers in connectomics.
\end{abstract}

\section{INTRODUCTION}

This book deals with the topics of connectomics and deep brain stimulation. But what is the connectome to begin with? How has the concept emerged and what are the current methods and approaches for mapping and studying it? In this chapter, we address these questions.

To understand the behavior of a complex system, we need to not only understand how the elements of that system behave in isolation, but how those elements interact with one another and the repertoire of patterns that can emerge from their collective interactions [1 3 . The human mind is one such complex system. It helps us sense and perceive our environment, create (and sometimes forget) memories, and even plays a role in fostering creativity. These types of behaviors are underpinned by our nervous system, which is composed of cells, neuronal populations, and brain areas [4. Yet, the mind does not emerge from these individual neural elements. Rather, complex behavior emerges from the distributed anatomical and functional networks created by interacting neural elements. To understand the mind, we must first understand the structure and function of brain networks 5 .

Historically, neuroscience has focused on properties of nervous systems that can be localized to individual neural elements, such as a cortical region's blood-oxygen-leveldependent (BOLD) activity or its curvature and gyrification. Recently, however, the emphasis has shifted toward studying properties of distributed networks 6 . This change in perspective is due in large part to the maturation of network science, which has provided a framework for mathematical representing and analyzing highdimensional datasets [7, 8] and has been applied successfully in other disciplines 913 .

These two events, along with data-sharing initiatives and access to high-performance computers, helped create a "connectomics revolution", in which neuroscience (and especially human neuroimaging using MRI methods) began to explicitly study structure and function of

\footnotetext{
* rbetzel@ indiana.edu
}

the brain from a network perspective [14, 15. Today, the procedures for generating brain networks have been largely automated [16, and network analyses are addressing fundamental questions in neuroscience concerning the brain's organization [17, and how it functions in health [18 21] and disease 22 24, with exquisite detail and personalization [25, 29].

In this chapter, we cover the basic tenets of connectomics and network neuroscience, focusing on large-scale human neuroimaging. We first introduce the concepts of functional and structural connectivity and outline the procedures for reconstructing these types of data from observations. We then discuss how these data can be modeled as a complex network, emphasizing three ways that network models of biological neural networks have revealed key insight into brain organization or function: structure-function relationships, brain network dynamics, and the brain's system-level architecture. Finally, we cover several frontier topics within connectomics, focusing on generative models, network controllability, and edge-centric approaches.

\section{STRUCTURAL AND FUNCTIONAL BRAIN NETWORKS}

Brain networks are maps that tells us which neural elements are connected, which are not, and how strong or efficacious each connection is [30]. Although these maps can be created at all spatial scales 31, 32, from cells to regions, connectomics has been embraced and proven especially transformative at the macroscale [15], where the smallest possible neural elements are voxels, each of which contains roughly $10^{6}$ neurons (although in practice, voxels get grouped together into larger parcels or regions of interest according to some set of shared features $33[38$ ). What does it mean for two regions or parcels in the brain to interact with one another [39]? In general, the field has converged on three different "flavors" or modalities by which two parts of the brain can interact with one another [40. 

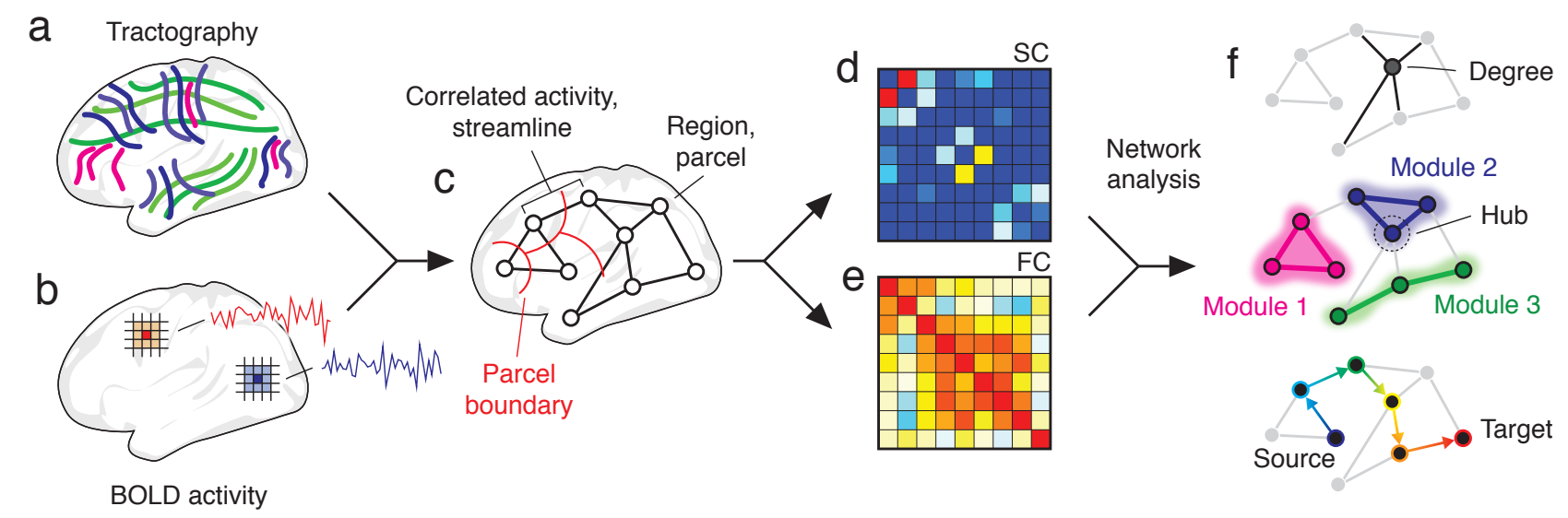

FIG. 1. Constructing, representing, and analyzing brain networks. Brain networks are typically constructed from diffusion-weighted and functional MRI data. (a) In the case of diffusion MRI, tractography is used to infer white-matter pathways. (b) In the case of functional MRI, connectivity is usually defined as the statistical dependence between BOLD time series recorded from voxels or surface vertices. (c) The brain is usually divided into parcels or regions so that each parcel is internally homogeneous and composed of voxels/vertices with similar sets of features. Region-by-region structural and functional connectivity matrices ( $d$ and $e$ ) are generated by aggregating connections weights according to parcels. In general, structural connectivity (SC) is sparse and its connections are weighted by streamline count, fractional anisotropy, or related measures. Functional connectivity (FC) is fully-weighted and, in the case of Pearson correlations, signed. $(f)$ The network representation of SC and FC can be subsequently analyzed, e.g. using network measures at local, meso-, and global scales.

\section{Flavors of connectivity}

The first measure of interactivity is anatomical or structural connectivity (SC), which describes the physical and material pathways of the brain. At the microand meso-scales, SC reflects synaptic coupling between cells or long-distance axonal projections between neuronal populations [41, 42]. At the macroscale, SC corresponds to large, myelinated white-matter fiber bundles [43. These bundles can be reconstructed from diffusion weighted MRI data, which measures the apparent direction in which water molecules diffuse in the brain, using a procedure known as "tractography," with many software packages capable of implementing this procedure efficiently (See Chapter 11) 44-47] (Fig. 17).

Effectively, SC restricts which neural elements can "talk" with one another, shaping signaling patterns and the flow of information throughout and inducing statistical dependencies between pairs of neural elements and their recorded activity 48, 49. These dependencies are referred to as functional connectivity (FC), in principle, can be quantified using any bivariate measure of statistical similarity [39, 40, 50,52] (Fig. 1b). These include spectral or wavelet coherence, mutual information, partial correlations, penalized correlations, to name a few. In practice, however, FC between brain regions is almost always measured as the bivariate (Pearson) correlation of the their activity when using functional MRI data (see Chapters 10 and 16).

There are, of course, other ways of measuring the inter- activity among neural elements. These include effective connectivity (EC) [53, 54, which offers methods like dynamic causal modeling [55, Granger causality [56], and transfer entropy [57, which measure the directed influence of one neural element on another. In general, estimating true causal relationships between neural elements connectivity is challenging 58 and requires experimental manipulation, e.g. observing the effect of a perturbation on the activity of other neural elements, or a specific model of how activity is relayed from one part of the brain to another, usually constrained by SC. Invasive procedures such as deep brain stimulation are especially promising in the context of EC, as they present a unique opportunity to map causal relationships between neural elements [59] and to validate existing causal models.

Other less-common methods for estimating the strength of interactions between neural elements include their structural covariance [60, which measures population-level similarity of regions morphological characteristics (although recent advances have made it possible to estimate similar metrics for single subjects [61]).

\section{Representing brain connectivity}

Irrespective of connection modality, in order to analyze patterns of brain connectivity, we need a way of representing those patterns mathematically. To do this, we turn to graph theory - a branch of mathematics concerned with the study of networks [17, 62. As briefly 
outlined in Chapter 1, graphs are mathematical representations of a network and consists of two essential ingredients: a set of vertices or nodes that represent the elementary units of a system, and a set of edges or connections that represent interactions between pairs of nodes. In a social system, e.g. an online community such as Twitter, nodes represent users and edges indicate whether one user follows another. In the case of the brain, nodes and edges usually represent brain regions and their structural or functional connections (Fig. 1. F). Although the simplest version of a graph is binary and undirected - i.e. where edges all have the same weight and encode symmetric relationships - graphs can also encode information about the weights and asymmetries of connections (those that are and are not reciprocated).

Irrespective of whether a network is weighted/binary, directed/undirected, the pairwise relationships between its nodes can be represented as an adjacency or connectivity matrix. The number of rows and columns in this square matrix is equal to the number of nodes in the network, and its elements represent the presence or absence and weights connections. In an SC matrix, weights usually correspond to streamline counts (the number of reconstructed streamlines between pairs of parcels), their density, or microstructural properties of streamlines, e.g. their fractional anisotropy (Fig. 1 $\mathrm{d}$ ). Although the precise density of connections in an SC matrix can vary as a function of processing pipelines, structural networks are generally sparse, meaning that of the connections that could exist, relatively few are actually present. Functional connections, as noted earlier, represent bivariate measures of statistical dependence and result in a fully weighted and signed matrix (Fig. 1p).

Care needs to be taken in preparing connectivity matrices - the procedures for inferring functional and SC are noisy and prone to both false positives and negatives. It is common, then, as a post-processing step, to impose some threshold on observed connections to retain those with the strongest weights or those least likely to represent false positives 63 67. It is worth noting, however, that thresholding is, in general, not a necessity; in some cases, it may be advantageous to retain all connections. In any case, the decision to threshold or not is non-trivial and will have implications for subsequent analysis of the network 68. In either case, the thresholded or unthresholded connectivity matrices can then be analyzed further using graph-theoretic network measure to summarize different aspects of a network's organization [17, 62, (Fig. 11). In the next section, we review some of these measures.

\section{NETWORK MEASURES AND ANALYSIS}

A network's adjacency matrix provides a succinct description of its topology - the configuration of connections among nodes. Analyzing brain networks usually involves studying connectivity matrices at different topo- logical scales, ranging from local measurements of individual brain regions and connections to global properties that characterize the entire brain. These measures are usually interpreted in terms of network function. In this section, we describe some of the more common measurements that can be made on a connectivity matrix and provide some interpretations.

\section{Local measures}

Local measures describe properties of individual nodes and edges. For instance, a node's degree simply counts the number of incoming and outgoing connections that the node makes (Fig. 1f). Despite its simplicity, a node's degree is one of the most fundamental measures that can be calculated for a network, influencing other local properties. Nodes with many connections - putative "hub" regions [69, 70] - tend to occupy positions of influence and import within a network (see Chapter 1 for how this may relate to deep brain stimulation).

Centrality measures quantify the importance of a node or an edge with respect to dynamical process taking place on a network or to other structural features of a network [71. The most common of these measures is betweenness centrality, which is calculated as the number of shortest paths between pairs of nodes that include a given node. Shortest paths in a network, which are discussed in more detail in the next section, are the shortest sequence of "hops" from a source node to a target node in a network. Betweenness centrality can also be calculated for network edges by tallying how many shortest paths involve a given connection. Of course there are other centrality measures that measure the importance of nodes and edges with respect to other processes and structure of a network, e.g. random walks [72] and communities [73].

Importantly, most local measures can be calculated for both binary, weighted, directed, and undirected networks [74. For instance, the weighted analog of node degree is node strength, which measures the total weight of all connections incident upon a given node. Similarly, a node's betweenness centrality can be computed based on shortest weighted paths between nodes.

\section{Global measures}

At the opposite extreme are global measures, which quantify properties of the network as a whole. A network's shortest paths structure, for instance, can be used to calculate several common global network measures including characteristic path length, which measures the average number of steps in shortest paths [75] (Fig. 1:). This measure has important functional consequences in transportation networks, where goods or information gets delivered from one node to another, shorter paths mean increased efficacy of transmission. In the context of a nervous system, where brain regions need to commu- 
nicate with one another, shorter paths mean faster transit times and fewer opportunities for a signal to degrade, get transformed, or otherwise attenuate [76, 77.

Characteristic path length is an unbounded measure, making it difficult to compare across networks of different sizes. It can also yield a value of $\infty$ if a network is composed of disconnected components (the path length between two disconnected nodes is infinite). To circumvent these issues, one can calculate a the related global measure of efficiency [78. Whereas characteristic path length is the average shortest path between all nodes, efficiency is equal to the average reciprocal shortest path length (1 divided by the length of the shortest path). Consequently, any nodes that are disconnected and have infinite path length now have an efficiency of $0(1 / \infty)$. The efficiency measure is bounded to the interval of $[0,1]$.

Importantly, both path length and efficiency can be extended from binary networks - where shortest paths correspond to number of hops or steps - to weighted networks. Some care needs to be taken, however. The algorithms used to map shortest paths seek to minimize a cost function - the number of steps or total weight along a path. However, connection weights in brain networks usually measure the affinity or strength of connection the most efficacious path from a seed to a target is the one that uses strongly-weighted connections. Consequently, shortest weighted paths can be calculated, but require the intermediary step of transforming connection weights from a measure of affinity to length. This is usually accomplished by a negative exponentiation of edge weights, i.e. $L_{i j}=W_{i j}^{-\gamma}$, or by dividing edge weights by their maximum value and taking the negative logarithm, i.e.

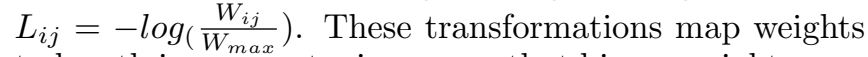
to length in a monotonic way, so that bigger weights necessarily correspond to smaller lengths. See 79 for an example of how such global network measures have been used to study effects of deep brain stimulation.

\section{Mesoscale measures}

Situated between the local and global scales is a rich mesoscale [80 82. Whereas local and global network properties describe features of nodes and edges or the network as a whole, mesoscale measures focus on properties of subnetworks within the larger network, which are thought to support specialized brain function (Fig. 1f). In the parlance of network science, these subnetworks or clusters are referred to as modules or "communities," in reference to social networks where communities represent groups of individuals. Analysis of mesoscale structure is arguably one of the most active areas of network science 83 (and of connectomics, as well 84), where it is used for pattern discovery, identification of functional groups, coarse-graining of networks, and for generating additional local and global network statistics.

However, with the exception of trivial examples, a network's mesoscale structure is unknown ahead of time and cannot be determined from visual inspection alone. Instead, mesoscale structure is detected algorithmically using a suite of "community detection" algorithms [85]. These algorithms aim to partition a network's nodes or edges into communities, usually so that communities are non-overlapping (although this is not always true 86 88]). The most popular methods in network neuroscience are modularity maximization [89] and Infomap 90, which seek partitions of nodes that optimize their respective objective functions.

The mesoscale, itself, spans multiple organizational levels, from a division of the network into singleton communities (every node is its own community) to a partition in which the entire network is assigned to the same cluster. Methods like modularity maximization can be parametrically tuned to detect communities of specific sizes between these two limits 91] (recent versions of Infomap have a similar ability).

Once a network's community structure has been detected, it can be used to calculate additional local and global network measures. For instance, the objective function, $Q$, used to optimize modularity maximization can be used as an index of how segregated a network's communities are from one another 84, 92. Similarly, we can view communities from the perspective of individual nodes or brain regions, and consider how their connections are distributed within and between modules using measures like participation coefficient 93, which, along with its partner measure, module degree $z$-score, can be used to infer whether a node acts as a hub and whether its influence extends beyond its own module. For instance, nodes with large participation coefficients have connections that span span module boundaries and are positioned to mediate flows between modules [94].

\section{WHAT HAVE WE LEARNED?}

Network neuroscience and connectomics provide a quantitative framework for investigating the brain's organization from a network perspective. Studies employing these methods are beginning to reveal the organization of functional and structural brain networks, shedding light on their operation in health and disease. For instance, like most real-world networks, brains exhibit small-world architecture [76, 77, 95], meaning that they have high levels of local clustering to support specialized processing but also topological "shortcuts" to reduce the average length of shortest paths, supporting rapid transmission of information across the brain 95. Other studies have demonstrated that brain networks exhibit heterogeneous degree distributions, such that a small number of brain regions make many more connections than others [96. These putative "hub" nodes occupy positions of influence and are interlinked to one another, forming a so-called "rich-club" of highly-influential brain regions [97, 98. Interestingly, rich club nodes are distributed throughout the brain's functional systems, suggesting that these in- 
terconnected hubs are positioned to integrate information from different sub-systems and modalities [99].

While modern connectomics is multifaceted and includes many active areas of research, both applied and theoretical, in the following section we discuss three particular topics that have been especially influential. These include mapping the brain's organization into functionally specialized sub-systems, elucidating the link between structural and FC, and tracking changes in functional network architecture across time.

\section{System-level organization}

Most real-world networks exhibit some form of mesoscale structure, such that nodes and edges can be meaningfully partitioned into sub-networks, modules, and communities [80, 100]. The character of these communities can vary, however. For instance, a network's community structure can form a dense core and sparse periphery [101, or even exhibit disassortative, multipartite structure where sub-networks are comprised of nodes that are sparsely connected internally, but exhibit many strong connections between groups [102]. The most common type of meso-scale organization, however, is assortative modular structure, where nodes are arranged into sub-networks that are densely connected internally, but weakly connected to one another [89, 90. Indeed, modular structure is ubiquitous in real-world networks [82, 103] and the development of new methods for detecting and characterizing these types of communities remains an active area of study in network science, broadly [104 106.

Is the brain modular? Why would we expect the brain to exhibit modular organization? Do brains with modules have some advantage that amodular brains are lacking?

A key feature of modular networks is the near autonomy of their modules, which enables them to develop specialized cognitive and psychological functions [107-109]. The autonomy of modules from one another also has implications for network robustness [110. Suppose a brain were damaged in some way, e.g. as the result of stroke, traumatic brain injury, or due to a deep brain stimulation electrode. In a modular brain, the effect of this damage remains localized to the module in which it originated, impacting only the set of functions supported by that module, but otherwise preserving the broader set of cognitive functions. Additionally, modular structure buffers cryptic variation, rendering brains more evolvable and leading to improved fitness [111. It helps reduce total cost of wiring and supports a rich repertoire of dynamics [112] and leads to reductions in wiring cost [113.

Is there any empirical evidence supporting the hypothesis that brains are modular? An ever-growing number of studies have used clustering and community detection algorithms to uncover the modular structure of real-world structural and functional brain networks [37, 96, 114-
124. Of these studies, some of the most compelling evidence that the brain exhibits modular structure comes from the analysis of resting-state functional imaging data 94, 125. In these studies, FC is estimated from fMRI data obtained at rest and clustered - in the first study, the clustering algorithm Infomap [90 was applied to thresholded connectivity matrices, and in the second, a modified $k$-means algorithm was applied to $\mathrm{FC}$ that was fully-weighted and signed. As expected, these algorithms partitioned parts of the brain into modules. Clustering algorithms, however, can mislead in that they can detect clusters even in networks with no true cluster structure [126 128. To mitigate this issue, both studies crossvalidated their clusters by comparing clusters' the spatial extents with task activation profiles from previous studies. Surprisingly, the boundaries of modules at rest circumscribed the activation patterns, suggesting that the brain's modular structure subtends the same systems that support active cognitive processing.

In another elegant study, Crossley et al. 129] directly compared the community structure of resting state connectivity with task co-activation patterns. Using data from BrainMap [130, 131, a database that curates and reports activation locations from many studies, Crossley constructed a co-activation matrix where nodes were connected to one another if they were active under similar conditions. The authors then applied a community detection method to these two matrices, and found a close correspondence between the resulting modules. These observations further suggest that the brain's intrinsic modular structure delineates its functional systems.

While there is an agreed-upon correspondence between communities and the brain's intrinsic functional architecture, there remains many open questions. For instance, what are the "true" number of communities? Is there a one-to-one correspondence of communities to functional domains? Do all brain regions form modules? Are all modules segregated and assortative?

Addressing these questions remains an area of active research and one that has required, in some cases, methodological innovation. Take for instance the question concerning the "true" number of modules and their respective sizes. Many studies have reported that the brain's modular structure is hierarchical, with smaller communities nested inside of larger communities 120 , 132. Even empirical studies have reported divisions of the brain into clusters of vastly different sizes, from large task-positive/-negative bipartitions [133] to many finescaled sub-divisions of these systems [134. However, methods like Infomap and modularity maximization return a partition of the network into modules at a single scale and into a fixed set of communities. How do we reconcile this reality with the expectation that modules should appear hierarchical? One strategy is to employ multi-scale community detection methods. Infomap and modularity maximization both include free parameters that can be tuned to detect different sizes and numbers of communities 4, effectively resulting in a description 
of a network's community structure that prioritizes no single scale. Rather, it embraces the brain's inherent multi-scale and hierarchical organization [135].

Community-level analyses of both functional and structural brain networks represent an active area of research and methods development. New techniques like multi-layer modularity maximization [136] make it possible to simultaneously cluster data networks from multiple individuals [137, and imaging modalities, and at different points in time [138. Other methods even challenge the basic assumption that the brain' community structure is modular. Stochastic blockmodels make fewer assumptions about the character of communities (both Infomap and modularity maximization can detect only assortative communities), and if core-periphery or disassortative communities exist, are capable of detecting them. Several recent studies using blockmodels have detected non-assortative communities [139 144, showing that by incorporating these features into models, strengthen structure-function relationships and align more closely with patterns of gene co-expression than communities estimated using modularity maximization [145.

Meso-scale analyses are also being used to characterize individual differences in cognition [146 148], clinical status [149, 150], and to track developmental [151, 152] and lifespan changes in brain network structure [153 155]. For instance, a growing number of studies have linked the quality of modules - quantified using the modularity function - with behavioral, demographic, and cognitive variables, such as biological age, performance on cognitively demanding tasks. Others have used modularity as prognostic measure, for instance to predict the outcomes therapy and targeted interventions [156 158].

Others, still, have investigated how the brain's modular structure reconfigures when the brain is engaged in cognitively demanding tasks [20]. Converging evidence suggests that a key organizing principle of task-evoked modular structure is that, relative to rest, modules exhibit increased integration and reduced segregation [159162. That is, cognitively demanding tasks seem to require that brain modules coordinate with one another, forming strong cross-module links. Methods like stochastic blockmodels report consistent findings, with assortative and segregated modules at rest getting replaced by non-assortative structures like core-periphery structure that, in at least some cases, is task-specific [163.

In summary, investigating the brain's system-level organization from a network science and connectomics perspective has informed multiple domains of neuroscience, from theory to application. This topic remains a productive area of research, and has spurred along methods development and is being applied fruitfully to brain data at all spatial scales 164 166.

\section{Structural basis of activity and connectivity}

One of the central questions in biology concerns the relationship of an organism structure with the kinds of functions it can perform. In the context of network neuroscience we can ask a similar question - how does the brain's anatomical structure shape patterns of functional coupling between distant sites in the brain [167]? Intuitively, we think of anatomical connectivity as a set of constraints - some parts of the brain are directly coupled to one another via white matter or axonal projections, others are not. Those that are connected can readily exchange information with or signal to one another those that lack direct connections can only communicate indirectly, through multi-step pathways, thereby shaping the correlation structure of brain activity [168. This coupling of function to the underlying anatomy is a fundamental feature of brain networks that, when disrupted or perturbed, can give rise to maladaptive behavior and disease [169]. A disruption of structure-function coupling may be exactly what we should aim at retuning with deep brain stimulation (see Chapter 1).

Indeed, there is considerable evidence - both empirical and in silico demonstrating that brain SC is causally related to patterns of functional coupling. Some of the strongest evidence comes from lesion studies, where direct structural insults lead to acute changes in FC. One of the clearest examples is a study by O'Reilly et al [170], in which resting state $\mathrm{FC}$ was estimated for macaque monkeys before and after surgically removal the collosal fibers between the left and right hemispheres. This had the acute effect of reducing the magnitude of interhemispheric functional connections, presumably because those regions can not longer exchange information or synchronize their activity with one another.

Other evidence that SC shapes functional coupling comes from in silico studies, where $\mathrm{SC}$ is considered part of a networked dynamical system (Fig. 2a; also see Chapter 26 for an outlook of how these concepts are applied to the field of deep brain stimulation). The elements of a dynamical system - nodes in the network - have internal states that represent their level of activity, e.g. membrane potential or BOLD amplitude, and are free to evolve over time according to some internal dynamics. When these elements are coupled to one another i.e. as parts of a network - then the evolution of each element's state depends on the states of its neighbors. Consequently, the constraints imposed by SC induce correlations in the change of nodes' states across time, leading to observed patterns of FC (Fig. 2p).

These kinds of models often include biophysical parameters, e.g. "neural mass models" include populations of excitatory and inhibitory neurons, channel conductances, temporally lagged propagation of signals, etc.. Although the inclusion of these parameters helps preserves some neurobiological plausibility, it comes with a cost. First, solving the system of differential equations is computationally expensive, and can entail hours of run-time on 


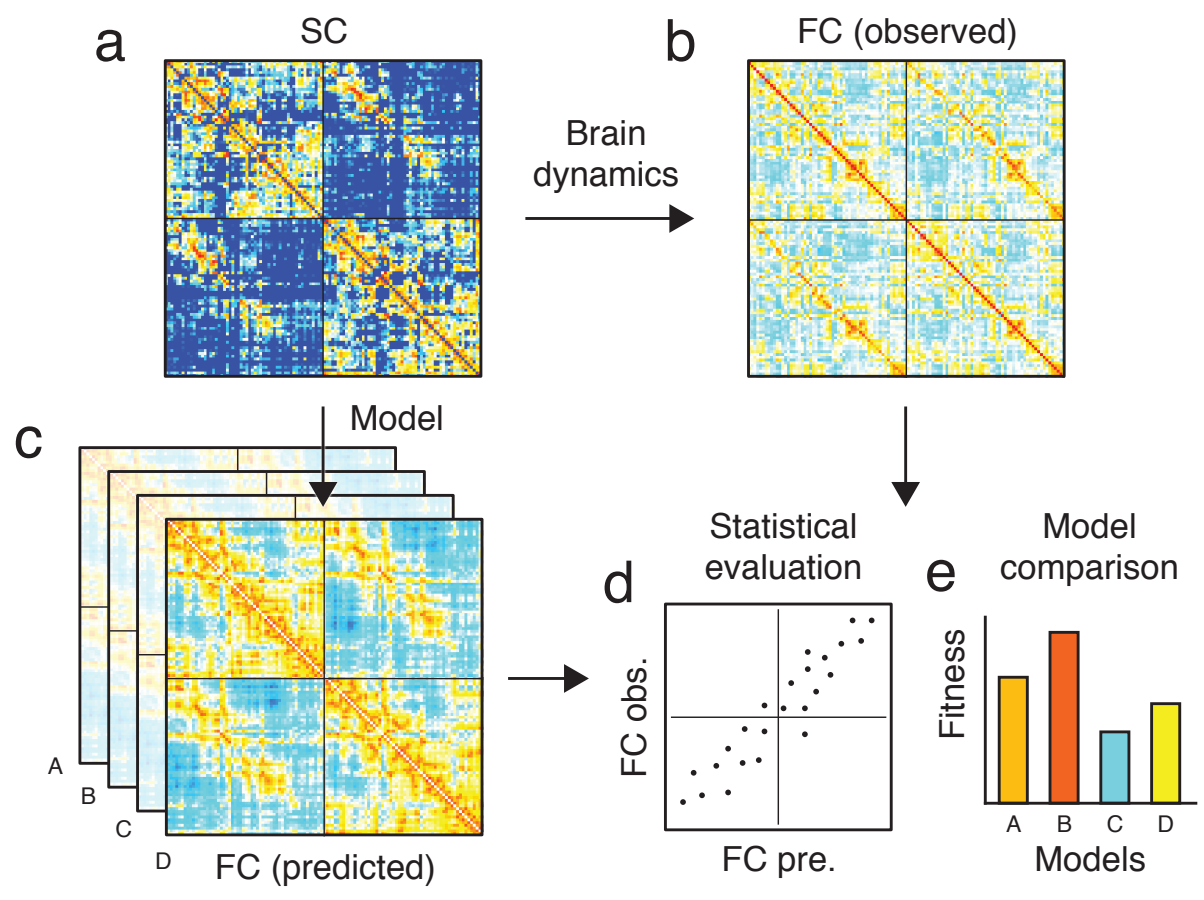

FIG. 2. Structurally constrained models of FC Structural connections $(a)$ constrain brain dynamics, which induces correlated brain activity that is measured as FC $(b)$. To better understand this structure-function relationship, one build models of brain dynamics that yield, as output, synthetic patterns of FC. (c) This framework makes it possible to test different models corresponding to distinct hypotheses about brain dynamics/communication with varying levels of complexity and biophysical plausibility. (d) Model performance can be quantified as a correlation between observed and predicted FC. (e) Models can be compared to one another using their performance as a measure of fitness to identify the optimal dynamics.

high-performance computers to generate even a few minutes of data (although new software packages promise to reduce the runtimes 171). Second, the correlation structure of the synthetic time series is only modestly similar to its empirical counterpart, suggesting that the biophysical models may be missing key components or parameters 48. This high computational cost for relatively low variance explained has prompted the exploration of simpler and more easily interpretable models based on analytically tractable, stylized dynamics that represent putative policies for interregional communication between brain regions [168].

Unlike the biophysical models, where the output is synthetic time series, communication models generate for all pairs of regions a measure of communication capacity or the ease with which two brain regions can communicate with one another, resulting in a square node-by-node matrix. For instance, suppose we hypothesize that brain regions "communicate" by routing signals through the network's shortest path structure. As a coarse test of this hypothesis, we could compute the length of shortest paths between all pairs of brain regions and compare the elements of the resulting shortest paths matrix with those of the empirical FC matrix. In general, stronger correspondence between the two matrices can be interpreted as evidence supporting the hypothesis that shortest paths are important for brain communication.

To take advantage of shortest paths for signaling, the "signals" would need to have global knowledge of the entire network and its shortest path structure. At the opposite extreme are decentralized processes like diffusion models in which a particle or random walker (representing some quanta of information) moves over the network randomly, hopping unbiased from one node to another [172 174]. From this process, one can compute measures like the mean first passage time that a particle starting at one node is expected to reach another, and compare these measures against FC. Other simple models include those based on communicability in which nodes communicate along all paths but discount longer paths exponentially [175], oscillator models where nodes update their phase based on those of their neighbors [176, and search information where paths between nodes are quantified based on their hiddenness (the amount of information necessary for a random walker to follow the shortest path without error) 49 . 
These simple models have allowed researchers to explore and compare a veritable zoo of possible communication policies, from multi-path communication 177 to models of epidemic spreading that have been repurposed for brain imaging data 178 180 to decentralized and greedy navigation models [181 183], to informationbased models [184, 185] (Fig. 22). Because they are based on analytically tractable measures, these models carry a low computational cost and can be easily implemented and their parameters optimized. Critically, these models tend to outperform the more neurobiologically plausible models in terms of how well they predict empirical FC [49] (Fig. 22, e).

Collectively, there is considerable empirical and in sil$i c o$ evidence suggesting that SC plays a causal role in shaping patterns of functional coupling. This evidence has prompted many studies to consider situations where normative structure-function coupling is perturbed. For example, during task performance [186], in neuropsychiatric disorders [187, or in human development [154, 188]. Indeed, variation in structure-function coupling has been associated with individual differences to performance on tasks, subject demographics, and traits [189.

There remain many open questions and challenges, however. For instance, the measured association between SC and FC likely depends on other factors, including the spatial embedding of the brain - the nearer two regions are to one another, the more likely they are to be connected by both modalities [190, 191]. This mutual dependence on distance makes assessing the true strength of structure-function coupling difficult. Other challenges are more fundamental. The veracity of SC and FC matrices remains a source of concern in most analyses. SC, especially, suffers from known biases that prevent accurate inference of certain white-matter tracts [192, 193. FC, too, exhibits a peculiar set of biases, including variation attributable to head motion in the scanner [194. More fundamentally, the de facto measure of functional coupling - the Pearson correlation - represents one of many possible measures of connectivity, and the strength of structure-function relationships will, in general, vary as a function of these measures.

\section{Connectome dynamics}

Among the profound realizations of the connectomics revolution is that brains are never quiet, even during cognitive rest 195. Rather, the human brain is in constant transit, traversing a high-dimensional landscape of activity and connectivity patterns over time. Time-varying changes in FC, in particular, have attracted considerable interest from the network neuroscience community [196], where it is generally accepted transient coupling and uncoupling of neural elements across time reflects changes in cognitive state and that by studying these changes, we can glean new insight into principles by which functional networks support psychological processes on fast timescales.

Many methods have been proposed to studying dynamic or time-varying FC [197, including frequencybased decompositions 198, instantaneous co-fluctuation analysis [199], and model-based approaches [200]. The most common, however, is the sliding-window approach 201. This entails specifying a "window" of some length comprising temporally contiguous time points (Fig. 3a,b). Then, temporally resolved FC is calculated using only those time points within a given window and the window advanced some fixed number of time points where the procedure gets repeated. The result is a sequence of networks corresponding to FC patterns at different points in time (Fig. 3F). These networks can then be analyzed to gain insight into network dynamics - how the topological organization of the brain fluctuates across time.

Although time-varying FC can be analyzed using many different approaches to quantify the stability and variability of patterns across time, one popular line of inquiry involves assigning time points to clusters based on the similarity FC patterns [202, 203]. This procedure yields a set of brain states - cluster centroids - that describe low-dimensional space that the brain traverses over time. These states can be summarized using a rich set of statistics that include the relative frequencies of each state and the probabilities of transitioning from one state to another. This approach has been used extensively and has helped link time-varying connectivity to arousal and wakefulness 204, 205, attention 206, and state of consciousness [207 209], and has been applied in a clinical context to generate biomarkers of disease and neuropsychiatric disorders [210.

Another powerful approach for studying time-varying connectivity is to track changes in communities across time using multi-layer network models 211] (Fig. 31). In this approach, FC patterns at different time points are treated as "layers" in a single multi-layer network, where each layer is weakly coupled to its temporally adjacent neighboring layers [136]. When the multi-layer network is clustered, all layers are clustered simultaneously and their cluster labels preserved. This makes it possible to directly compare nodes' cluster labels across temporally adjacent layers to identify flexible nodes that switch their cluster assignments frequently and those that maintain stable assignments across time 212. This approach can be used to track the formation, persistence, and dissolution of communities across time [138, 213]. Flexibility can calculated both at the whole-brain and regional levels and in previous studies has proven to be a potent biomarker of cognition and disease. A growing number of studies have linked patterns of flexibility with learning [138, 214, 215], executive function [216], mood and affective state [217], and working memory [218. Other studies have linked flexibility to psychiatric disorders and schizophrenia 219.

Although analyses of time-varying FC using the sliding window approach is common, this procedure requires 


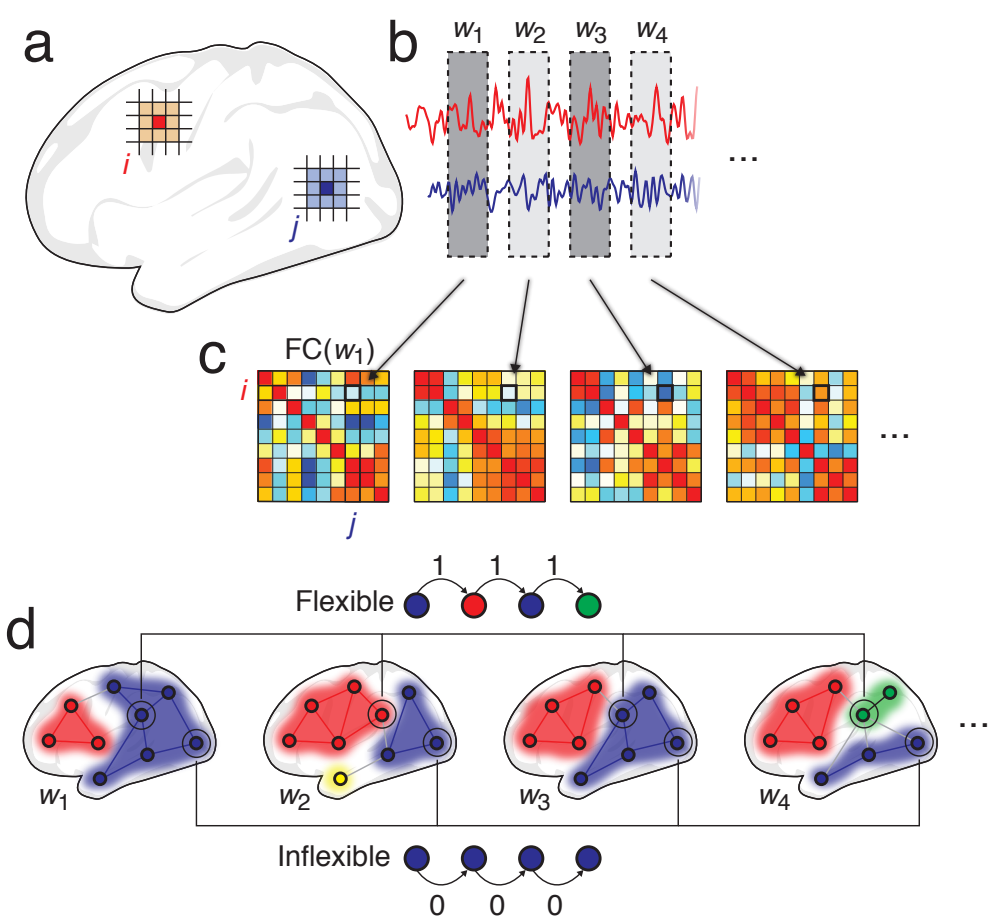

FIG. 3. Time-varying FC and flexibility. To study changes in network organization across time, sliding window methods are routinely used to obtain time-varying estimates of FC. (a) This involves extracting time courses from different parts of the brain. (b) Time series are then "windowed" and FC weights are estimated using only the points that fall within a given window. For a pair of voxels/vertices/parcels, this results in a sequence of FC estimates that are temporally localized to that window. $(c)$ This procedure can be repeated for all pairs of brain regions, resulting in a time series of FC matrices. (d) Although time-varying FC can be analyzed using many different methods, one common approach is based on reconfiguration of network modules to estimate network flexibility. This procedure involves constructing a "multi-layer network" from the time-varying matrices and clustering all time points simultaneously. Flexibility is calculated as the frequency with which a node changes its modular affiliation from one time point to the next. Here, we show examples of a node that is flexible (top) and another that is inflexible (bottom).

the user to make several non-trivial processing decisions along. These include deciding upon window length and whether it should be tapered or not 220] and how far the window should be advanced at each step [221]. Although varying the length of is a simple way to study network changes at different timescales, window length also has an impact on sampling variability. This leads to a tradeoff between timescale and errors, where shorter windows can reveal faster time-varying changes in FC but with a greater proportion of false positives and negatives [222, 223.

Other issues challenge the very notion that timevarying FC could be measured using fMRI methods. These studies argue that, due to the slow and indirect measures obtained using fMRI, the variability observed in time-varying FC analyses is consistent with the sampling variability from a temporally stationary and unchanging correlation structure [224, 225]. This observation necessitates that any estimate of time-varying connectivity be compared against a stationary null model.

In summary, time-varying network analyses are being applied widely to study changes in network structure over shorter timescales. The results of these analyses are providing insight into the principles by which brain networks reconfigure across time. Work in this area has proven controversial at times 226, but continues to strengthen the link between fast changes in network topology and fluctuations in cognitive state.

\section{WHAT DOES THE FUTURE HOLD?}

Like any young field, the full potential of connectomics and network neuroscience remains untapped, with exciting new work taking place on many frontiers. In this section, we review several of these areas, focusing on topics of generative modeling, network control, and edge-centric approaches. 

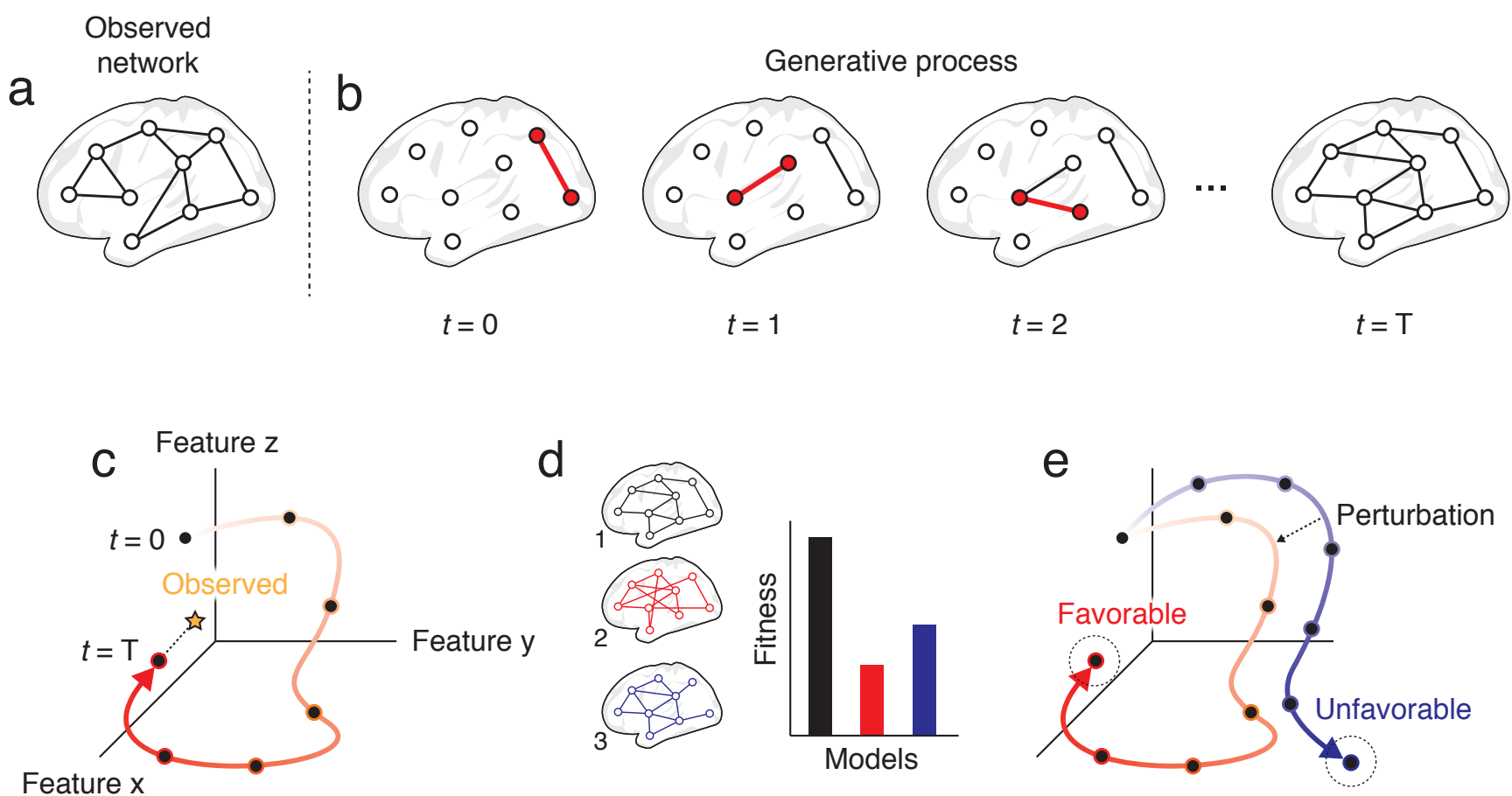

FIG. 4. Generative models of connectomes. Network generative models aim to uncover the wiring rules that give rise to an observed brain network $(a)$. (b) This is accomplished by proposing and testing different models - generative processes - and evaluating how well they match features of the observed network. The generative processes can be stylized and take place on non-biological timescales or they can incorporate detailed neurophysiological details and take place over developmental time. (c) This process traces a trajectory through a state space, where the outcome - a synthetic brain network - is compared to the observed network using some measure of distance/similarity. The generative modeling framework allows for in silico explorations of interventions. For instance, if two networks evolve on similar trajectories - one to a favorable, healthy state and the other to an unfavorable, disease state - one can use explore targeted perturbations of the unfavorable trajectory to identify strategies that would "drive" the brain back towards the healthy state. (e) Different generative models that test distinct hypotheses about network growth and evolution can be instantiated and their fitness - how well they match features of the observed network - can be compared to gain insight into the wiring rules and principles that shape brain network organization.

\section{Generative modeling}

The appeal of network neuroscience stems in part from the ability to quantify different aspects of brain organization using an ever-growing suite of summary statistics. As noted earlier, these measures can diagnose influential nodes, discover communities, and assess a network's capacity for efficient information transmission. However, network summary statistics are just that - they describe and summarize a network. That is, summary statistics offer no explanation for why a network has a certain feature or how that feature came to exist in the first place. To understand the processes, rules, and algorithms by which networks grow and evolve requires building, testing, and validating generative models 227.

Generative modeling has a rich history in network science with the foundational Watts-Strogatz 95] and Barabasi-Albert models 228 models serving as the clearest examples. While these models posit mechanisms by which small-worldness and hubs emerge, in general, gen- erative models work by identifying a desired set of properties for a network to have, and work backwards to identify the processes that yields networks with those properties (Fig. 4 a,b,c,d).

As with other modeling exercises, parsimony plays an important role, and generative modeling studies usually aim to discover "simple" wiring rules that can accurately replicate properties of real brain networks. These rules, then, can be interpreted as drivers of the brain's network organization. Any additional features that emerge incidentally and as a consequence of these rules can be thought of as "spandrels" and useful byproducts of the overarching generative mechanism [229].

The space of possible generative models for brain networks is massive. One way that we can impose some order on this space to categorize models according to how they deal with time. On one extreme are models in which the network grows and evolves over a biologically meaningful timescale, with the aim of recapitulating a specific developmental trajectory. These kinds of models need to 
be carefully calibrated against observations and include other neurobiological details, but can then be used to assess how perturbations alter developmental trajectories (leading to maladaptive brain networks) and to explore strategies for driving a brain back onto a healthy trajectory, all in silico (Fig. 4 ). A good example of this type of model is the work by Nicosia et al [230] that modeled the growth of the nematode, $C$. elegans. $C$. elegans is unique in that we have detailed information about the birth times of its roughly 300 neurons, which can then be incorporated into a generative growth model with the aim of reproducing the network of the adult $C$. elegans. The authors demonstrated that a model based on birth times combined and wiring rules that penalize the formation of long connections and reward nodes with high degree could account for many of the properties of the adult $C$. elegans connectome, including bi-phasic growth rate.

At the opposite extreme are models for which time plays no role, like stochastic blockmodels, or deal with time in a non-biological way. The Barabasi-Albert model is a good example, in that the network grows according to a set of simple rules, adding nodes and edges over a series of steps, but where the timescale is arbitrary. While these types of models lack biological realism, they can be used to gain insight into the underlying principles that shape network organization. For instance, several recent network neuroscience studies have investigated quasi-dynamic models, in which edges are gradually added to a set of brain regions according to a probabilistic growth rule [231, 232]. In these studies, the authors tested many possible rules based on the spatial layout of the network and its topology, comparing their abilities to account for organizational features of empirical brain networks. In both cases, the authors found that the optimal model - the one that consistently produced synthetic networks with features similar to realworld brain networks - penalized the formation of longdistance connections but increased the likelihood of connections forming between nodes with similar connectivity profiles. Although the timescale of these models lacked any correspondence with neurodevelopment, their results were consistent with the generative model of $C$. elegans.

Generative models have been instrumental in uncovering potential organizing principles of brain networks. Among the key findings is the role played by spatial relationships in shaping the brain's network structure [233235. Brains must be enclosed in a limited volume and their connections require material to form and energy to maintain and use. Shorter connections, therefore, lead to reductions in volume and wiring cost, and lead to increased fitness. Although some studies maintain that the drive to reduce these costs is sufficient to explain brain network organization in its entirety, most agree that this drive needs to be counter-balanced by some opposing force to account for costly features of brain networks that involve long-distance connections, e.g. the presence of hubs and rich-clubs 236 238.
The generative modeling approach opens up new, exciting strategies for studying brain networks and for understanding their roles in health and disease. First, generative models help discover the drivers of network organization, which helps refocus attention onto those features and away from "spandrels" [229], which may help in the generation of increasingly sensitive and appropriate biomarkers. Second, generative models can be fit to subject-specific data and their parameters used to study individual differences. For instance, in 232. the authors demonstrated the parameters governing spatial constraints varied with age, while [238] used similar models and demonstrated that their parameters were correlated with polygenic risk of schizophrenia and cognitive performance. Lastly, generative models can provide insight into human development by incorporating additional neurobiological and developmental details into their wiring rules [239].

\section{Network control}

In order to meet ongoing cognitive demands, the human brain must seamlessly transition from one brain state to another in order. How does the brain accomplish this? How are these transitions supported by the underlying anatomical connectivity? These types of questions can be addressed using the network control framework 240 242]. This approach presupposes that the brain is a networked dynamical system and that, in the absence of any intervention, the activity of each brain region evolves over time according to its own state and the states of connected neighbors, tracing out a trajectory over time. However, this trajectory can be altered by exogenous time-varying input signals that are "injected" into brain region. As a result, these signals can drive brain activity, causing it to deviate from its passive trajectory. Network control theory asks the question of whether its possible to tailor these input signals so that instead of simply following a different trajectory, the brain is directed along a specific, predefined trajectory using the lowest amplitude control signals possible.

In this way, network control offers an elegant and mathematically tractable framework that naturally links brain connectivity, dynamics, and activity. On one hand, it can be used to yield a set of local and global network measures. These include brain region's control profiles - their abilities to drive the brain into certain classes of brain states, e.g. those that are hard to reach and require significant amounts of effort to reach versus those that are easier to reach, requiring little effort 243 245]. We can also compute global measures that reveal whether a network is or is not controllable - i.e. whether its topology and dynamics allow it to be driven into any arbitrary state. Like other network measures, control metrics can be calculated for individual subjects and used to study inter-individual or group-level differences [246248, and have been used to reveal changes across devel- 
opment [249, 250], differences between control and clinical populations 251, and to understand that structural and dynamic underpinnings of creative ability [252].

In reality, most brain states are never visited. That is, they correspond to patterns of activity that represent non-functional "noise" or are actively dangerous to visit, e.g. seizure or epileptic activity. To make control measures more informative and realistic, one should focus exclusively on transitions between brain states that are actively visited, and ignore those that, for one reason or another, are not. One way to do this is to leverage the optimal control framework. Instead of considering transitions to and from all possible states, the optimal control framework considers transitions between specific pairs of states: the initial state in which the system begins and the target state that it is trying to reach. Given a set of control nodes through which input signals are injected, optimal control delivers the time-varying inputs that drive the brain from the initial state to its target using as little effort as possible. The amount of effort - the control energy - depends on the character of the initial and target states, but also the topology of the underlying structural network [253 256]. The optimal control framework yields a series of statistics that can be compared across individuals or between groups. These include nodal trajectories, input signals, and the control energy needed to support the transition. These measures have been used in several applied contexts. For instance, 257] calculated brain states as clusters of brain activity patterns, and demonstrated that the most frequent state transitions required the smallest amounts of energy, suggesting that the brain's white-matter network supports its dynamic trajectory through state space. Similarly, 258] computed the energy required to transition to and from activity states during a working memory task, and linked the amount of energy to expression of dopamine receptors.

On one hand, the very premise of network control that we can selectively drive the brain into different patterns of activity with distinct cognitive and behavioral analogs - seems like science fiction 259. On the contrary - network control theory has real-world applications and practical implications. For instance, with the advent of implantable neuromodulatory devices, the widespread use of non-invasive stimulation (e.g. transcranial magnetic stimulation), and the practice of optogenetic control of population-level activity, new theory is necessary to model and predict the effect of targeted stimulation. Nonetheless, there remain some practical hurdles to using network control. To date, most applications of network control to large-scale brain networks assume that brain activity is described by a linear dynamics. While the assumption helps simplify the mathematics of control 245, linear [21], controlling non-linear dynamics is considerably more challenging than linear control [260, 261]. A second issue concerns the relationship of controllability measures with more easily obtained, traditional network measures, e.g. nodal degree. Several articles have demonstrated that these measures are highly co-linear, suggesting that what determines a node's control profile is not the topology of the network, low-level features of nodes, e.g. the number of connections they make. The implication is that the control profiles of a real and randomized network would be nearly identical [262, 263].

Nonetheless, network control represents an exciting new frontier in connectomics. It opens up the tantalizing possibility of selectively driving whole-brain patterns of activity using knowledge of the underlying structural connections. While the framework is mostly relegated to exercises in theory, new methods for manipulating brain activity, which include deep brain stimulation, will provide cases where theory can be carefully tested and further refined.

\section{Edge-centric connectomics}

Historically, brain networks have been modeled and analyzed using "node-centric" networks whose the nodes and edges represent neurons, populations, or areas, and functional or structural connections. This model has been the workhorse of network neuroscience and is at the core of virtually every connectomics discovery to date. Recently, however, several groups have proposed extending the node-centric model to characterize higher-order interactions in a network [88, 264, 265]. This means shifting focus away from interactions between brain regions and neural elements, and building altogether new classes of networks that focus on the interactions between functional and structural connections.

The two most commonly discussed approaches for generating higher-order "edge-centric" networks involve transforming traditional node-by-node connectivity matrices into higher-dimensional edge-by-edge "line graphs" 264] and "edge similarity" matrices [88 that quantify how strongly pairs of edges in the network interact with one another. While these approaches have been applied successfully in other disciplines, their application in neuroscience has been limited. To date, only one study has applied edge-centric methods to empirical brain networks, using line graphs to study the overlapping community structure of SC data [266].

Recently, however, several papers presented a new method for generating higher-order networks, in the process making them easier to apply to neuroimaging data and opening up new opportunities for studying the brain's higher-order organization and dynamics 267 271. The new edge-centric model is based on time series and the bivariate Pearson correlation - the measure routinely used to quantify FC. Whereas the Pearson correlation of two regions' activity is calculated as the mean element-wise product of their standardized (zscored) time series (Fig. 5a,b), the edge-centric model omits the averaging step, yielding a co-fluctuation time series for every pair of nodes (edge). Because FC is often interpreted as an index of communication between two 


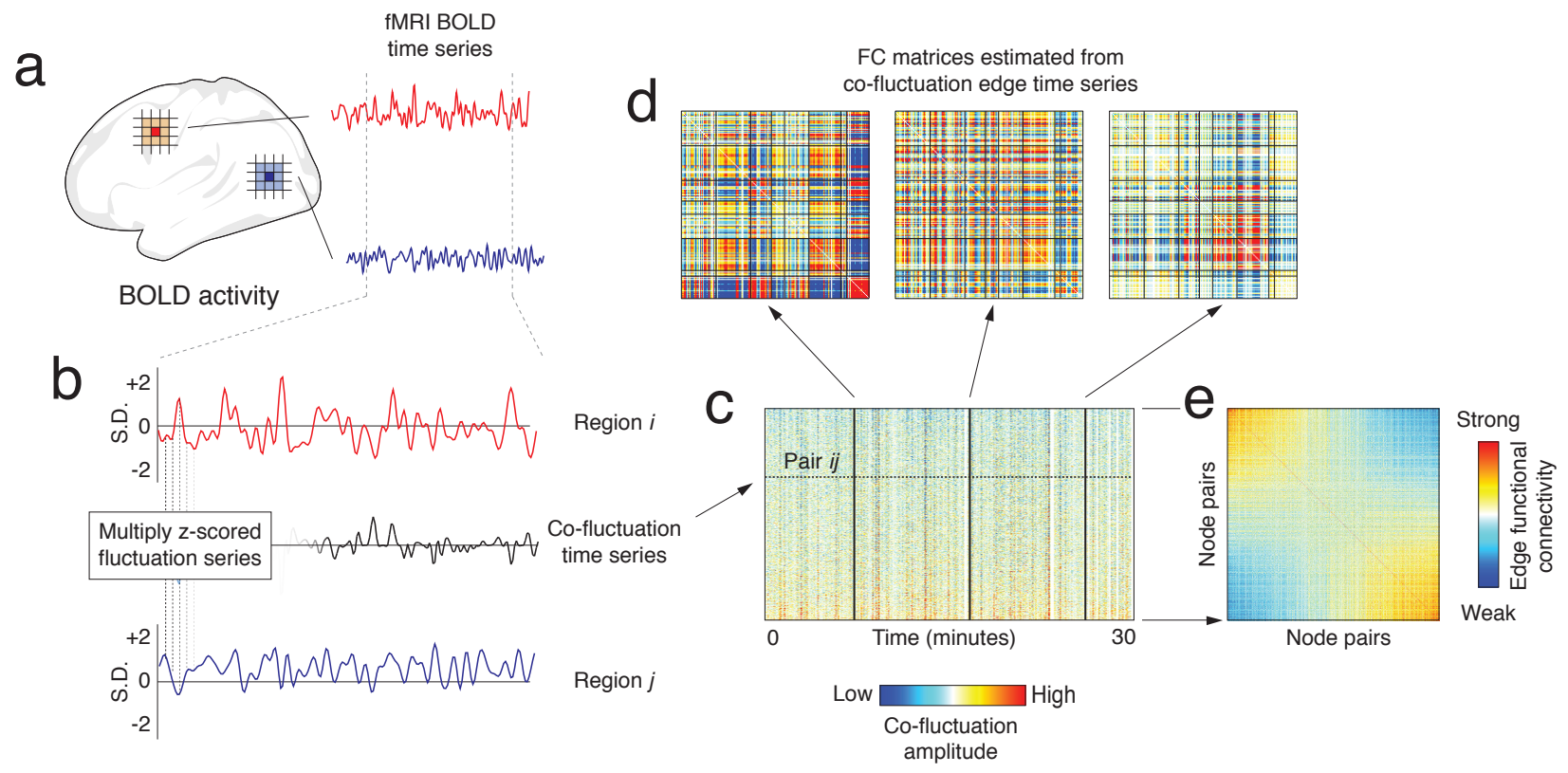

FIG. 5. Edge-centric modeling and analysis of neuroimaging data. Edge-centric models shift focus away from individual brain regions and their activity onto pairs of regions and their coactivity. (a) This is accomplished by first extracting and zscoring regional/parcel time series, which are incidentally the first two steps in computing fFC. (b) For every pair of time series, we compute the element-wise product of their activity, resulting in a co-activity or co-fluctuation time series. (c) This procedure is carried out for all pairs of brain regions, yielding an matrix of "edge" time series. ( $d$ ) Each column of the edge time series matrix represents a pattern of co-activity expressed at a specific instant. These patterns can be reshaped into a region-by-region matrix and analyzed as a functional network. (e) Edge time series can also be used to create a novel type of connectivity matrix: "edge functional connectivity" (eFC). This is accomplished by computing the pairwise similarity between all possible pairs of edge time series. Unlike nodal FC, which results in a region-by-region matrix, the eFC matrix has dimensions of node pairs by node pairs, i.e. edge by edge. This matrix can be analyzed further, e.g. clustered to reveal overlapping communities.

brain regions, we can think of each edge time series as a time-resolved account of that "conversation".

This procedure can be carried out for every pair of brain regions - all the edges in the network - to yield a complete set of co-fluctuation time series (also referred to as "edge time series"), which has useful properties (Fig. 5F). First, we can view edge time series as a temporal decomposition of $\mathrm{FC}$; the temporal average of the co-fluctuation time series for any pair of nodes is exactly the weight of the functional connection between those nodes. With this decomposition, then, we can assess the contribution to average FC of individual frames. Additionally, if we "slice" edge time series at a given point in time we obtain the instantaneous co-fluctuation pattern for all pairs of edges, which can be reshaped into a regionby-region matrix and analyzed as a network (Fig. 5d). Thus, edge time series quantify time-varying changes in network structure without using a sliding window, resulting in improved temporal resolution.

Interestingly, edge time series appear bursty - i.e. the amplitude is weak at most points in time, but over very short intervals becomes extremely strong [268. Even more interesting, the times at which these bursts occur tend to be correlated across edges, resulting timevarying networks where most connections are weak, but are occasionally punctuated by rare and short-lived highamplitude "events." Although events occur infrequently, they explain a disproportionate amount of variance in static FC, account for its system-level structure, and contain subject-specific information not present in loweramplitude frames.

Edge time series can also be used to estimate the higher-order construct of edge functional connectivity (eFC). eFC is obtained by computing the similarity between pairs of edge time series, yielding an edge-by-edge connectivity matrix analogous to the line graphs and link similarity networks but is fully weighted and signed [88, 264] (Fig. 5e). Like traditional node-centric networks, eFC can be analyzed using tools from network science to identify important or influential edges in the network. The principal advantage of eFC, however, is when community detection algorithms are used to partition edges into communities of cohesively fluctuating edge time series. In this context, edges are assigned to a single community, but because each node is affiliated with many edges, each of which can belong to a different community, nodes in the network can have multiple community affiliations. Unlike other methods that resolve overlapping communities in which a small fraction of nodes participate weakly in multiple communities 
[272, 273], edge communities result in "pervasive overlap," such that nodes are tiled by multiple overlapping communities. This notion of pervasive overlap agrees with our understanding of brain function; rather than subtending a narrow or singular set of functions, even traditionally "unimodal" brain areas respond to a range of diverse stimuli and conditions [274, 275]. In Faskowitz et al 267, entropy measures were used to quantify the level of overlap in each brain region, which revealed heterogeneous patterns. Interestingly, the highest levels of overlap were observed in sensorimotor and attentional networks, suggesting that these regions may play a previously undisclosed role in supporting a wide range of cognitive functions.

In summary, the edge-centric approach provides another lens through which we can study the brain's organization, function, and dynamics. Just as SC and FC offer complementary insight, so does edge connectivity, revealing changes in co-fluctuation patterns over fast timescales and characterizing the brain's higher-order interactions. The edge-centric approach presents multiple opportunities for future studies. First, we can take advantage of the fact that edge time series decompose FC into its framewise contributions to reconstruct FC using only select subsets of frames. These subsets can be chosen in a specific way so as to maximize the relationship of the reconstructed FC with behavior or to enhance group differences. eFC, itself, appears to be useful in amplifying subject-specific features of networks [270, opening up the possibility that this added personalization will im- prove studies of individual differences.

\section{CONCLUSION}

A perfect confluence of factors - availability of data, analytic framework, and computational resources - has created an environment that allows network neuroscience and connectomics to flourish. These new disciplines provide a quantitative framework for exploring patterns of brain connectivity in health and disease and across a vast range of spatiotemporal scales. This framework is beginning to reveal some of the key features of brain networks - small-world architecture for efficient information processing, modules to support specialized information processing, hubs and rich clubs for integrating information between modules, and a strong dependence on spatial layout that helps reduce the brain's material and metabolic cost of wiring. The future of connectomics includes powerful new methodologies for uncovering network-level mechanisms and controlling brain activity while new recording and imaging techniques make it possible to resolve networks at increasingly finer spatial scales but with the benefit of broader brain coverage. While the advanced concepts introduced here are necessarily new and continue to be explored and refined within the field of network neuroscience proper, timevarying connectivity, network control, and edge-centric connectomics hold promise in applied fields, such as connectomic deep brain stimulation. It is likely, then, that in future studies, these and other cutting edge methods from network neuroscience will move from exercises in theory and into application.
[1] Danielle S Bassett and Michael S Gazzaniga, "Understanding complexity in the human brain," Trends in cognitive sciences 15, 200-209 (2011).

[2] Hae-Jeong Park and Karl Friston, "Structural and functional brain networks: from connections to cognition," Science 342 (2013).

[3] Olaf Sporns, "The human connectome: a complex network," Annals of the New York Academy of Sciences 1224, 109-125 (2011).

[4] Richard F Betzel and Danielle S Bassett, "Multi-scale brain networks," Neuroimage 160, 73-83 (2017).

[5] Danielle S Bassett and Olaf Sporns, "Network neuroscience," Nature neuroscience 20, 353-364 (2017).

[6] Olaf Sporns and Danielle S Bassett, "New trends in connectomics," (2018).

[7] Mark Ed Newman, Albert-László Ed Barabási, and Duncan J Watts, The structure and dynamics of networks. (Princeton university press, 2006).

[8] Albert-László Barabási et al., Network science (Cambridge university press, 2016).

[9] Stanley Wasserman and Joseph Galaskiewicz, Advances in social network analysis: Research in the social and behavioral sciences (Sage, 1994).
[10] Hawoong Jeong, Sean P Mason, A-L Barabási, and Zoltan N Oltvai, "Lethality and centrality in protein networks," Nature 411, 41-42 (2001).

[11] Albert-László Barabási, Natali Gulbahce, and Joseph Loscalzo, "Network medicine: a network-based approach to human disease," Nature reviews genetics 12, 56-68 (2011).

[12] Marc Vidal, Michael E Cusick, and Albert-László Barabási, "Interactome networks and human disease," Cell 144, 986-998 (2011).

[13] Dmitri Krioukov, Maksim Kitsak, Robert S Sinkovits, David Rideout, David Meyer, and Marián Boguñá, "Network cosmology," Scientific reports 2, 793 (2012).

[14] Bharat B Biswal, Maarten Mennes, Xi-Nian Zuo, Suril Gohel, Clare Kelly, Steve M Smith, Christian F Beckmann, Jonathan S Adelstein, Randy L Buckner, Stan Colcombe, et al., "Toward discovery science of human brain function," Proceedings of the National Academy of Sciences 107, 4734-4739 (2010).

[15] R Cameron Craddock, Saad Jbabdi, Chao-Gan Yan, Joshua T Vogelstein, F Xavier Castellanos, Adriana Di Martino, Clare Kelly, Keith Heberlein, Stan Colcombe, and Michael P Milham, "Imaging human con- 
nectomes at the macroscale," Nature methods 10, 524 (2013).

[16] Oscar Esteban, Christopher J Markiewicz, Ross W Blair, Craig A Moodie, A Ilkay Isik, Asier Erramuzpe, James D Kent, Mathias Goncalves, Elizabeth DuPre, Madeleine Snyder, et al., "fmriprep: a robust preprocessing pipeline for functional mri," Nature methods 16, 111-116 (2019).

[17] Ed Bullmore and Olaf Sporns, "Complex brain networks: graph theoretical analysis of structural and functional systems," Nature reviews neuroscience 10, 186198 (2009).

[18] Malaak Nasser Moussa, Crystal D Vechlekar, Jonathan H Burdette, Matt $\mathrm{R}$ Steen, Christina E Hugenschmidt, and Paul J Laurienti, "Changes in cognitive state alter human functional brain networks," Frontiers in human neuroscience 5, 83 (2011).

[19] William R Shirer, Srikanth Ryali, Elena Rykhlevskaia, Vinod Menon, and Michael D Greicius, "Decoding subject-driven cognitive states with whole-brain connectivity patterns," Cerebral cortex 22, 158-165 (2012).

[20] Michael W Cole, Danielle S Bassett, Jonathan D Power, Todd S Braver, and Steven E Petersen, "Intrinsic and task-evoked network architectures of the human brain," Neuron 83, 238-251 (2014).

[21] Michael W Cole, Takuya Ito, Danielle S Bassett, and Douglas H Schultz, "Activity flow over resting-state networks shapes cognitive task activations," Nature neuroscience 19, 1718-1726 (2016).

[22] Cedric Huchuan Xia, Zongming Ma, Rastko Ciric, Shi $\mathrm{Gu}$, Richard F Betzel, Antonia N Kaczkurkin, Monica E Calkins, Philip A Cook, Angel Garcia de la Garza, Simon N Vandekar, et al., "Linked dimensions of psychopathology and connectivity in functional brain networks," Nature communications 9, 1-14 (2018).

[23] Ang Li, Andrew Zalesky, Weihua Yue, Oliver Howes, Hao Yan, Yong Liu, Lingzhong Fan, Kirstie J Whitaker, Kaibin $\mathrm{Xu}$, Guangxiang Rao, et al., "A neuroimaging biomarker for striatal dysfunction in schizophrenia," Nature Medicine 26, 558-565 (2020).

[24] Alex Fornito, Andrew Zalesky, and Michael Breakspear, "The connectomics of brain disorders," Nature Reviews Neuroscience 16, 159-172 (2015).

[25] Timothy O Laumann, Evan M Gordon, Babatunde Adeyemo, Abraham Z Snyder, Sung Jun Joo, MeiYen Chen, Adrian W Gilmore, Kathleen B McDermott, Steven M Nelson, Nico UF Dosenbach, et al., "Functional system and areal organization of a highly sampled individual human brain," Neuron 87, 657-670 (2015).

[26] Russell A Poldrack, Timothy O Laumann, Oluwasanmi Koyejo, Brenda Gregory, Ashleigh Hover, Mei-Yen Chen, Krzysztof J Gorgolewski, Jeffrey Luci, Sung Jun Joo, Ryan L Boyd, et al., "Long-term neural and physiological phenotyping of a single human," Nature communications 6, 1-15 (2015).

[27] Russell A Poldrack, "Precision neuroscience: Dense sampling of individual brains," Neuron 95, 727-729 (2017).

[28] Evan M Gordon, Timothy O Laumann, Adrian W Gilmore, Dillan J Newbold, Deanna J Greene, Jeffrey J Berg, Mario Ortega, Catherine Hoyt-Drazen, Caterina Gratton, Haoxin Sun, et al., "Precision functional mapping of individual human brains," Neuron 95, 791-807 (2017).
[29] Caterina Gratton, Timothy O Laumann, Ashley N Nielsen, Deanna J Greene, Evan M Gordon, Adrian W Gilmore, Steven M Nelson, Rebecca S Coalson, Abraham Z Snyder, Bradley L Schlaggar, et al., "Functional brain networks are dominated by stable group and individual factors, not cognitive or daily variation," Neuron 98, 439-452 (2018).

[30] Olaf Sporns, Giulio Tononi, and Rolf Kötter, "The human connectome: a structural description of the human brain," PLoS Comput Biol 1, e42 (2005).

[31] Manuel Schröter, Ole Paulsen, and Edward T Bullmore, "Micro-connectomics: probing the organization of neuronal networks at the cellular scale," Nature Reviews Neuroscience 18, 131-146 (2017).

[32] Martijn P Van den Heuvel, Edward T Bullmore, and Olaf Sporns, "Comparative connectomics," Trends in cognitive sciences 20, 345-361 (2016).

[33] Simon B Eickhoff, BT Thomas Yeo, and Sarah Genon, "Imaging-based parcellations of the human brain," Nature Reviews Neuroscience 19, 672-686 (2018).

[34] Alexander Schaefer, Ru Kong, Evan M Gordon, Timothy O Laumann, Xi-Nian Zuo, Avram J Holmes, Simon B Eickhoff, and BT Thomas Yeo, "Local-global parcellation of the human cerebral cortex from intrinsic functional connectivity mri," Cerebral cortex 28, 30953114 (2018).

[35] Lingzhong Fan, Hai Li, Junjie Zhuo, Yu Zhang, Jiaojian Wang, Liangfu Chen, Zhengyi Yang, Congying Chu, Sangma Xie, Angela R Laird, et al., "The human brainnetome atlas: a new brain atlas based on connectional architecture," Cerebral cortex 26, 3508-3526 (2016).

[36] Rahul S Desikan, Florent Ségonne, Bruce Fischl, Brian T Quinn, Bradford C Dickerson, Deborah Blacker, Randy L Buckner, Anders M Dale, R Paul Maguire, Bradley T Hyman, et al., "An automated labeling system for subdividing the human cerebral cortex on mri scans into gyral based regions of interest," Neuroimage 31, 968-980 (2006).

[37] Evan M Gordon, Timothy O Laumann, Babatunde Adeyemo, Jeremy F Huckins, William M Kelley, and Steven E Petersen, "Generation and evaluation of a cortical area parcellation from resting-state correlations," Cerebral cortex 26, 288-303 (2016).

[38] Matthew F Glasser, Timothy S Coalson, Emma C Robinson, Carl D Hacker, John Harwell, Essa Yacoub, Kamil Ugurbil, Jesper Andersson, Christian F Beckmann, Mark Jenkinson, et al., "A multi-modal parcellation of human cerebral cortex," Nature 536, 171-178 (2016).

[39] Barry Horwitz, "The elusive concept of brain connectivity," Neuroimage 19, 466-470 (2003).

[40] Karl J Friston, "Functional and effective connectivity in neuroimaging: a synthesis," Human brain mapping 2, 56-78 (1994).

[41] Seung Wook Oh, Julie A Harris, Lydia Ng, Brent Winslow, Nicholas Cain, Stefan Mihalas, Quanxin Wang, Chris Lau, Leonard Kuan, Alex M Henry, et al., "A mesoscale connectome of the mouse brain," Nature 508, 207-214 (2014).

[42] Nikola T Markov, MM Ercsey-Ravasz, AR Ribeiro Gomes, Camille Lamy, Loic Magrou, Julien Vezoli, P Misery, A Falchier, R Quilodran, MA Gariel, et al., "A weighted and directed interareal connectivity matrix for macaque cerebral cortex," 
Cerebral cortex 24, 17-36 (2014).

[43] Peter J Basser, Sinisa Pajevic, Carlo Pierpaoli, Jeffrey Duda, and Akram Aldroubi, "In vivo fiber tractography using dt-mri data," Magnetic resonance in medicine 44, 625-632 (2000).

[44] Alessandro Daducci, Stephan Gerhard, Alessandra Griffa, Alia Lemkaddem, Leila Cammoun, Xavier Gigandet, Reto Meuli, Patric Hagmann, and JeanPhilippe Thiran, "The connectome mapper: an opensource processing pipeline to map connectomes with mri," PloS one 7, e48121 (2012).

[45] J-Donald Tournier, Fernando Calamante, and Alan Connelly, "Mrtrix: diffusion tractography in crossing fiber regions," International journal of imaging systems and technology 22, 53-66 (2012).

[46] FC Yeh, "Diffusion mri reconstruction in dsi studio," Advanced Biomedical MRI Lab, National Taiwan University Hospital. Available online at: http://dsistudio. labsolver. org/Manual/Reconstruction\# TOCQ-Space-Diffeomorphic-Reconstruction-QSDR (2017).

[47] Matthew Cieslak, Philip A Cook, Xiaosong He, FangCheng Yeh, Thijs Dhollander, Azeez Adebimpe, Geoffrey K Aguirre, Danielle S Bassett, Richard F Betzel, Josiane Bourque, et al., "Qsiprep: An integrative platform for preprocessing and reconstructing diffusion mri," bioRxiv (2020).

[48] Christopher J Honey, Olaf Sporns, Leila Cammoun, Xavier Gigandet, Jean-Philippe Thiran, Reto Meuli, and Patric Hagmann, "Predicting human resting-state functional connectivity from structural connectivity," Proceedings of the National Academy of Sciences 106, 2035-2040 (2009).

[49] Joaquín Goñi, Martijn P Van Den Heuvel, Andrea Avena-Koenigsberger, Nieves Velez De Mendizabal, Richard F Betzel, Alessandra Griffa, Patric Hagmann, Bernat Corominas-Murtra, Jean-Philippe Thiran, and Olaf Sporns, "Resting-brain functional connectivity predicted by analytic measures of network communication," Proceedings of the National Academy of Sciences 111, 833-838 (2014).

[50] Dongli Zhou, Wesley K Thompson, and Greg Siegle, "Matlab toolbox for functional connectivity," Neuroimage 47, 1590-1607 (2009).

[51] Stephen M Smith, Karla L Miller, Gholamreza SalimiKhorshidi, Matthew Webster, Christian F Beckmann, Thomas E Nichols, Joseph D Ramsey, and Mark W Woolrich, "Network modelling methods for fmri," Neuroimage 54, 875-891 (2011).

[52] Usama Pervaiz, Diego Vidaurre, Mark W Woolrich, and Stephen M Smith, "Optimising network modelling methods for fmri," NeuroImage 211, 116604 (2020).

[53] Karl J Friston, "Functional and effective connectivity: a review," Brain connectivity 1, 13-36 (2011).

[54] Karl Friston, Rosalyn Moran, and Anil K Seth, "Analysing connectivity with granger causality and dynamic causal modelling," Current opinion in neurobiology 23, 172-178 (2013).

[55] Klaas Enno Stephan, Will D Penny, Rosalyn J Moran, Hanneke EM den Ouden, Jean Daunizeau, and Karl J Friston, "Ten simple rules for dynamic causal modeling," Neuroimage 49, 3099-3109 (2010).

[56] Anil K Seth, Adam B Barrett, and Lionel Barnett, "Granger causality analysis in neuroscience and neuroimaging," Journal of Neuroscience 35, 3293-3297
(2015).

[57] Thomas Schreiber, "Measuring information transfer," Physical review letters 85, 461 (2000).

[58] Ioana E Marinescu, Patrick N Lawlor, and Konrad P Kording, "Quasi-experimental causality in neuroscience and behavioural research," Nature human behaviour 2, 891-898 (2018).

[59] Diana H Lim, Majid H Mohajerani, Jeffrey LeDue, Jamie Boyd, Shangbin Chen, and Timothy $\mathrm{H}$ Murphy, "In vivo large-scale cortical mapping using channelrhodopsin-2 stimulation in transgenic mice reveals asymmetric and reciprocal relationships between cortical areas," Frontiers in neural circuits 6, 11 (2012).

[60] Andrea Mechelli, Karl J Friston, Richard S Frackowiak, and Cathy J Price, "Structural covariance in the human cortex," Journal of Neuroscience 25, 8303-8310 (2005).

[61] Jakob Seidlitz, František Váša, Maxwell Shinn, Rafael Romero-Garcia, Kirstie J Whitaker, Petra E Vértes, Konrad Wagstyl, Paul Kirkpatrick Reardon, Liv Clasen, Siyuan Liu, et al., "Morphometric similarity networks detect microscale cortical organization and predict inter-individual cognitive variation," Neuron 97, 231247 (2018).

[62] Mikail Rubinov and Olaf Sporns, "Complex network measures of brain connectivity: uses and interpretations," Neuroimage 52, 1059-1069 (2010).

[63] Andrew Zalesky, Alex Fornito, Luca Cocchi, Leonardo L Gollo, Martijn P van den Heuvel, and Michael Breakspear, "Connectome sensitivity or specificity: which is more important?" Neuroimage 142, 407-420 (2016).

[64] James A Roberts, Alistair Perry, Gloria Roberts, Philip B Mitchell, and Michael Breakspear, "Consistency-based thresholding of the human connectome," Neuroimage 145, 118-129 (2017).

[65] Martijn P van den Heuvel, Siemon C de Lange, Andrew Zalesky, Caio Seguin, BT Thomas Yeo, and Ruben Schmidt, "Proportional thresholding in restingstate fmri functional connectivity networks and consequences for patient-control connectome studies: Issues and recommendations," Neuroimage 152, 437-449 (2017).

[66] Richard F Betzel, Alessandra Griffa, Patric Hagmann, and Bratislav Mišić, "Distance-dependent consensus thresholds for generating group-representative structural brain networks," Network neuroscience 3, 475-496 (2019).

[67] Fabrizio De Vico Fallani, Vito Latora, and Mario Chavez, "A topological criterion for filtering information in complex brain networks," PLoS computational biology 13, e1005305 (2017).

[68] Kathleen A Garrison, Dustin Scheinost, Emily S Finn, Xilin Shen, and R Todd Constable, "The (in) stability of functional brain network measures across thresholds," Neuroimage 118, 651-661 (2015).

[69] Olaf Sporns, Christopher J Honey, and Rolf Kötter, "Identification and classification of hubs in brain networks," PloS one 2, e1049 (2007).

[70] Martijn P van den Heuvel and Olaf Sporns, "Network hubs in the human brain," Trends in cognitive sciences 17, 683-696 (2013).

[71] Xi-Nian Zuo, Ross Ehmke, Maarten Mennes, Davide Imperati, F Xavier Castellanos, Olaf Sporns, and Michael P Milham, "Network centrality in the human functional connectome," Cerebral cortex 22, 1862-1875 
(2012).

[72] Phillip Bonacich, "Factoring and weighting approaches to status scores and clique identification," Journal of mathematical sociology 2, 113-120 (1972).

[73] Ernesto Estrada and Juan A Rodriguez-Velazquez, "Subgraph centrality in complex networks," Physical Review E 71, 056103 (2005).

[74] Tore Opsahl, Filip Agneessens, and John Skvoretz, "Node centrality in weighted networks: Generalizing degree and shortest paths," Social networks 32, 245-251 (2010).

[75] Mark EJ Newman, "Scientific collaboration networks. ii. shortest paths, weighted networks, and centrality," Physical review E 64, 016132 (2001).

[76] Olaf Sporns and Jonathan D Zwi, "The small world of the cerebral cortex," Neuroinformatics 2, 145-162 (2004).

[77] Sophie Achard, Raymond Salvador, Brandon Whitcher, John Suckling, and ED Bullmore, "A resilient, lowfrequency, small-world human brain functional network with highly connected association cortical hubs," Journal of Neuroscience 26, 63-72 (2006).

[78] Vito Latora and Massimo Marchiori, "Efficient behavior of small-world networks," Physical review letters 87, 198701 (2001).

[79] Tim J Van Hartevelt, Joana Cabral, Gustavo Deco, Arne Møller, Alexander L Green, Tipu Z Aziz, and Morten L Kringelbach, "Neural plasticity in human brain connectivity: the effects of long term deep brain stimulation of the subthalamic nucleus in parkinson's disease," PloS one 9, e86496 (2014).

[80] Mark EJ Newman, "Communities, modules and largescale structure in networks," Nature physics 8, 25-31 (2012).

[81] Mark EJ Newman, "Modularity and community structure in networks," Proceedings of the national academy of sciences 103, 8577-8582 (2006).

[82] Michelle Girvan and Mark EJ Newman, "Community structure in social and biological networks," Proceedings of the national academy of sciences 99, 7821-7826 (2002).

[83] Santo Fortunato and Darko Hric, "Community detection in networks: A user guide," Physics reports 659, 1-44 (2016).

[84] Olaf Sporns and Richard F Betzel, "Modular brain networks," Annual review of psychology 67, 613-640 (2016).

[85] Santo Fortunato, "Community detection in graphs," Physics reports 486, 75-174 (2010).

[86] Gergely Palla, Imre Derényi, Illés Farkas, and Tamás Vicsek, "Uncovering the overlapping community structure of complex networks in nature and society," nature 435, 814-818 (2005).

[87] Jierui Xie, Stephen Kelley, and Boleslaw K Szymanski, "Overlapping community detection in networks: The state-of-the-art and comparative study," Acm computing surveys (csur) 45, 1-35 (2013).

[88] Yong-Yeol Ahn, James P Bagrow, and Sune Lehmann, "Link communities reveal multiscale complexity in networks," nature 466, 761-764 (2010).

[89] Mark EJ Newman and Michelle Girvan, "Finding and evaluating community structure in networks," Physical review E 69, 026113 (2004).
[90] Martin Rosvall and Carl T Bergstrom, "Maps of random walks on complex networks reveal community structure," Proceedings of the National Academy of Sciences 105, 1118-1123 (2008).

[91] Jörg Reichardt and Stefan Bornholdt, "Statistical mechanics of community detection," Physical review E 74, 016110 (2006).

[92] Gagan S Wig, "Segregated systems of human brain networks," Trends in cognitive sciences 21, 981-996 (2017).

[93] Roger Guimera and Luis A Nunes Amaral, "Functional cartography of complex metabolic networks," nature 433, 895-900 (2005).

[94] Jonathan D Power, Alexander L Cohen, Steven M Nelson, Gagan S Wig, Kelly Anne Barnes, Jessica A Church, Alecia C Vogel, Timothy O Laumann, Fran M Miezin, Bradley L Schlaggar, et al., "Functional network organization of the human brain," Neuron 72, 665-678 (2011).

[95] Duncan J Watts and Steven H Strogatz, "Collective dynamics of "small-world'networks," nature 393, 440-442 (1998).

[96] Patric Hagmann, Leila Cammoun, Xavier Gigandet, Reto Meuli, Christopher J Honey, Van J Wedeen, and Olaf Sporns, "Mapping the structural core of human cerebral cortex," PLoS Biol 6, e159 (2008).

[97] Martijn P Van Den Heuvel and Olaf Sporns, "Richclub organization of the human connectome," Journal of Neuroscience 31, 15775-15786 (2011).

[98] Gorka Zamora-López, Changsong Zhou, and Jürgen Kurths, "Cortical hubs form a module for multisensory integration on top of the hierarchy of cortical networks," Frontiers in neuroinformatics 4, 1 (2010).

[99] Martijn P Van den Heuvel and Olaf Sporns, "An anatomical substrate for integration among functional networks in human cortex," Journal of Neuroscience 33, 14489-14500 (2013).

[100] Mason A Porter, Jukka-Pekka Onnela, and Peter J Mucha, "Communities in networks," Notices of the AMS 56, 1082-1097 (2009).

[101] M Puck Rombach, Mason A Porter, James H Fowler, and Peter J Mucha, "Core-periphery structure in networks," SIAM Journal on Applied mathematics 74, 167-190 (2014).

[102] Tsuyoshi Murata, "Detecting communities from bipartite networks based on bipartite modularities," in 2009 International Conference on Computational Science and Engineering, Vol. 4 (IEEE, 2009) pp. 50-57.

[103] Jure Leskovec, Kevin J Lang, Anirban Dasgupta, and Michael W Mahoney, "Community structure in large networks: Natural cluster sizes and the absence of large well-defined clusters," Internet Mathematics 6, 29-123 (2009).

[104] Till Hoffmann, Leto Peel, Renaud Lambiotte, and Nick S Jones, "Community detection in networks without observing edges," Science advances 6, eaav1478 (2020).

[105] Pan Zhang and Cristopher Moore, "Scalable detection of statistically significant communities and hierarchies, using message passing for modularity," Proceedings of the National Academy of Sciences 111, 18144-18149 (2014).

[106] Tiago P Peixoto, "Hierarchical block structures and high-resolution model selection in large networks," Physical Review X 4, 011047 (2014). 
[107] Herbert A Simon, "The architecture of complexity," in Facets of systems science (Springer, 1991) pp. 457-476.

[108] Carlos Espinosa-Soto and Andreas Wagner, "Specialization can drive the evolution of modularity," PLoS Comput Biol 6, e1000719 (2010).

[109] Jerry A Fodor, The modularity of mind (MIT press, 1983).

[110] Tien-Dzung Tran and Yung-Keun Kwon, "The relationship between modularity and robustness in signalling networks," Journal of The Royal Society Interface 10, 20130771 (2013).

[111] Marc Kirschner and John Gerhart, "Evolvability," Proceedings of the National Academy of Sciences 95, 84208427 (1998).

[112] Raj Kumar Pan and Sitabhra Sinha, "Modularity produces small-world networks with dynamical timescale separation," EPL (Europhysics Letters) 85, 68006 (2009).

[113] Danielle S Bassett, Daniel L Greenfield, Andreas MeyerLindenberg, Daniel R Weinberger, Simon W Moore, and Edward T Bullmore, "Efficient physical embedding of topologically complex information processing networks in brains and computer circuits," PLoS comput biol 6, e1000748 (2010).

[114] Richard F Betzel, Alessandra Griffa, Andrea AvenaKoenigsberger, Joaquín Goñi, Jean-Philippe Thiran, Patric Hagmann, and Olaf Sporns, "Multi-scale community organization of the human structural connectome and its relationship with resting-state functional connectivity," Network Science 1, 353-373 (2013).

[115] Kai Wu, Yasuyuki Taki, Kazunori Sato, Yuko Sassa, Kentaro Inoue, Ryoi Goto, Ken Okada, Ryuta Kawashima, Yong He, Alan C Evans, et al., "The overlapping community structure of structural brain network in young healthy individuals," PloS one 6, e19608 (2011).

[116] Malaak N Moussa, Matthew R Steen, Paul J Laurienti, and Satoru Hayasaka, "Consistency of network modules in resting-state fmri connectome data," PloS one 7, e44428 (2012).

[117] Jeanette A Mumford, Steve Horvath, Michael C Oldham, Peter Langfelder, Daniel H Geschwind, and Russell A Poldrack, "Detecting network modules in fmri time series: a weighted network analysis approach," Neuroimage 52, 1465-1476 (2010).

[118] Simon B Eickhoff, Danilo Bzdok, Angela R Laird, Christian Roski, Svenja Caspers, Karl Zilles, and Peter T Fox, "Co-activation patterns distinguish cortical modules, their connectivity and functional differentiation," Neuroimage 57, 938-949 (2011).

[119] David Meunier, Renaud Lambiotte, and Edward T Bullmore, "Modular and hierarchically modular organization of brain networks," Frontiers in neuroscience 4, 200 (2010)

[120] David Meunier, Renaud Lambiotte, Alex Fornito, Karen Ersche, and Edward T Bullmore, "Hierarchical modularity in human brain functional networks," Frontiers in neuroinformatics 3, 37 (2009).

[121] Richard F Betzel, John D Medaglia, Lia Papadopoulos, Graham L Baum, Ruben Gur, Raquel Gur, David Roalf, Theodore D Satterthwaite, and Danielle S Bassett, "The modular organization of human anatomical brain networks: Accounting for the cost of wiring," Network Neuroscience 1, 42-68 (2017).
[122] Maxwell A Bertolero, BT Thomas Yeo, and Mark D'Esposito, "The modular and integrative functional architecture of the human brain," Proceedings of the National Academy of Sciences 112, E6798-E6807 (2015).

[123] Teddy J Akiki and Chadi G Abdallah, "Determining the hierarchical architecture of the human brain using subject-level clustering of functional networks," Scientific reports 9, 1-15 (2019).

[124] Andrew C Murphy, Shi Gu, Ankit N Khambhati, Nicholas F Wymbs, Scott T Grafton, Theodore D Satterthwaite, and Danielle S Bassett, "Explicitly linking regional activation and function connectivity: community structure of weighted networks with continuous annotation," arXiv preprint arXiv:1611.07962 (2016).

[125] BT Thomas Yeo, Fenna M Krienen, Jorge Sepulcre, Mert R Sabuncu, Danial Lashkari, Marisa Hollinshead, Joshua L Roffman, Jordan W Smoller, Lilla Zöllei, Jonathan R Polimeni, et al., "The organization of the human cerebral cortex estimated by intrinsic functional connectivity," Journal of neurophysiology (2011).

[126] Roger Guimera, Marta Sales-Pardo, and Luís A Nunes Amaral, "Modularity from fluctuations in random graphs and complex networks," Physical Review E 70, 025101 (2004).

[127] Leto Peel, Daniel B Larremore, and Aaron Clauset, "The ground truth about metadata and community detection in networks," Science advances 3, e1602548 (2017).

[128] Darko Hric, Richard K Darst, and Santo Fortunato, "Community detection in networks: Structural communities versus ground truth," Physical Review E 90, 062805 (2014).

[129] Nicolas A Crossley, Andrea Mechelli, Petra E Vértes, Toby $\mathrm{T}$ Winton-Brown, Ameera X Patel, Cedric E Ginestet, Philip McGuire, and Edward T Bullmore, "Cognitive relevance of the community structure of the human brain functional coactivation network," Proceedings of the National Academy of Sciences 110, 1158311588 (2013).

[130] Angela R Laird, Jack J Lancaster, and Peter T Fox, "Brainmap," Neuroinformatics 3, 65-77 (2005).

[131] Peter T Fox and Jack L Lancaster, "Mapping context and content: the brainmap model," Nature Reviews Neuroscience 3, 319-321 (2002).

[132] Gaëlle Doucet, Mikaël Naveau, Laurent Petit, Nicolas Delcroix, Laure Zago, Fabrice Crivello, Gael Jobard, Nathalie Tzourio-Mazoyer, Bernard Mazoyer, Emmanuel Mellet, et al., "Brain activity at rest: a multiscale hierarchical functional organization," Journal of neurophysiology 105, 2753-2763 (2011).

[133] Yulia Golland, Polina Golland, Shlomo Bentin, and Rafael Malach, "Data-driven clustering reveals a fundamental subdivision of the human cortex into two global systems," Neuropsychologia 46, 540-553 (2008).

[134] Evan M Gordon, Timothy O Laumann, Scott Marek, Ryan V Raut, Caterina Gratton, Dillan J Newbold, Deanna J Greene, Rebecca S Coalson, Abraham Z Snyder, Bradley L Schlaggar, et al., "Default-mode network streams for coupling to language and control systems," Proceedings of the National Academy of Sciences 117, 17308-17319 (2020).

[135] Arian Ashourvan, Qawi K Telesford, Timothy Verstynen, Jean M Vettel, and Danielle S Bassett, "Multiscale detection of hierarchical community architecture 
in structural and functional brain networks," Plos one 14, e0215520 (2019).

[136] Peter J Mucha, Thomas Richardson, Kevin Macon, Mason A Porter, and Jukka-Pekka Onnela, "Community structure in time-dependent, multiscale, and multiplex networks," science 328, 876-878 (2010).

[137] Richard F Betzel, Maxwell A Bertolero, Evan M Gordon, Caterina Gratton, Nico UF Dosenbach, and Danielle S Bassett, "The community structure of functional brain networks exhibits scale-specific patterns of inter-and intra-subject variability," Neuroimage 202, 115990 (2019).

[138] Danielle S Bassett, Nicholas F Wymbs, Mason A Porter, Peter J Mucha, Jean M Carlson, and Scott T Grafton, "Dynamic reconfiguration of human brain networks during learning," Proceedings of the National Academy of Sciences 108, 7641-7646 (2011).

[139] Dragana M Pavlovic, Petra E Vértes, Edward T Bullmore, William R Schafer, and Thomas E Nichols, "Stochastic blockmodeling of the modules and core of the caenorhabditis elegans connectome," PloS one 9, e97584 (2014).

[140] Daniel Moyer, Boris Gutman, Gautam Prasad, Joshua Faskowitz, Greg Ver Steeg, and Paul Thompson, "Blockmodels for connectome analysis," in 11th International Symposium on Medical Information Processing and Analysis, Vol. 9681 (International Society for Optics and Photonics, 2015) p. 96810A.

[141] Joshua Faskowitz, Xiaoran Yan, Xi-Nian Zuo, and Olaf Sporns, "Weighted stochastic block models of the human connectome across the life span," Scientific reports 8, 1-16 (2018).

[142] Joshua Faskowitz and Olaf Sporns, "Mapping the community structure of the rat cerebral cortex with weighted stochastic block modeling," Brain Structure and Function 225, 71-84 (2020).

[143] Dragana M Pavlović, Bryan RL Guillaume, Emma K Towlson, Nicole MY Kuek, Soroosh Afyouni, Petra E Vértes, BT Thomas Yeo, Edward T Bullmore, and Thomas E Nichols, "Multi-subject stochastic blockmodels for adaptive analysis of individual differences in human brain network cluster structure," NeuroImage , 116611 (2020).

[144] Shi Gu, Cedric Huchuan Xia, Rastko Ciric, Tyler M Moore, Ruben C Gur, Raquel E Gur, Theodore D Satterthwaite, and Danielle S Bassett, "Unifying the notions of modularity and core-periphery structure in functional brain networks during youth," Cerebral Cortex 30, 1087-1102 (2020).

[145] Richard F Betzel, John D Medaglia, and Danielle S Bassett, "Diversity of meso-scale architecture in human and non-human connectomes," Nature communications 9, 1-14 (2018).

[146] Alexander A Stevens, Sarah C Tappon, Arun Garg, and Damien A Fair, "Functional brain network modularity captures inter-and intra-individual variation in working memory capacity," PloS one 7, e30468 (2012).

[147] Pauline L Baniqued, Courtney L Gallen, Michelle W Voss, Agnieszka Z Burzynska, Chelsea N Wong, Gillian E Cooke, Kristin Duffy, Jason Fanning, Diane K Ehlers, Elizabeth A Salerno, et al., "Brain network modularity predicts exercise-related executive function gains in older adults," Frontiers in aging neuroscience 9, 426 (2018).
[148] Maxwell A Bertolero, BT Thomas Yeo, Danielle S Bassett, and Mark D'Esposito, "A mechanistic model of connector hubs, modularity and cognition," Nature human behaviour 2, 765-777 (2018).

[149] Aaron F Alexander-Bloch, Nitin Gogtay, David Meunier, Rasmus Birn, Liv Clasen, Francois Lalonde, Rhoshel Lenroot, Jay Giedd, and Edward T Bullmore, "Disrupted modularity and local connectivity of brain functional networks in childhood-onset schizophrenia," Frontiers in systems neuroscience 4, 147 (2010).

[150] Ye He, Sol Lim, Santo Fortunato, Olaf Sporns, Lei Zhang, Jiang Qiu, Peng Xie, and Xi-Nian Zuo, "Reconfiguration of cortical networks in mdd uncovered by multiscale community detection with fmri," Cerebral Cortex 28, 1383-1395 (2018).

[151] Shi Gu, Theodore D Satterthwaite, John D Medaglia, Muzhi Yang, Raquel E Gur, Ruben C Gur, and Danielle S Bassett, "Emergence of system roles in normative neurodevelopment," Proceedings of the National Academy of Sciences 112, 13681-13686 (2015).

[152] Graham L Baum, Rastko Ciric, David R Roalf, Richard F Betzel, Tyler M Moore, Russell T Shinohara, Ari E Kahn, Simon N Vandekar, Petra E Rupert, Megan Quarmley, et al., "Modular segregation of structural brain networks supports the development of executive function in youth," Current Biology 27, 1561-1572 (2017).

[153] Micaela Y Chan, Denise C Park, Neil K Savalia, Steven E Petersen, and Gagan S Wig, "Decreased segregation of brain systems across the healthy adult lifespan," Proceedings of the National Academy of Sciences 111, E4997-E5006 (2014).

[154] Richard F Betzel, Lisa Byrge, Ye He, Joaquín Goñi, Xi-Nian Zuo, and Olaf Sporns, "Changes in structural and functional connectivity among resting-state networks across the human lifespan," Neuroimage 102, 345-357 (2014).

[155] Linda Geerligs, Remco J Renken, Emi Saliasi, Natasha M Maurits, and Monicque M Lorist, "A brainwide study of age-related changes in functional connectivity," Cerebral cortex 25, 1987-1999 (2015).

[156] Katelyn L Arnemann, Anthony J-W Chen, Tatjana Novakovic-Agopian, Caterina Gratton, Emi M Nomura, and Mark D'Esposito, 'Functional brain network modularity predicts response to cognitive training after brain injury," Neurology 84, 1568-1574 (2015).

[157] Courtney L Gallen, Pauline L Baniqued, Sandra B Chapman, Sina Aslan, Molly Keebler, Nyaz Didehbani, and Mark D'Esposito, "Modular brain network organization predicts response to cognitive training in older adults," PloS one 11, e0169015 (2016).

[158] Courtney L Gallen and Mark D'Esposito, "Brain modularity: a biomarker of intervention-related plasticity," Trends in cognitive sciences 23, 293-304 (2019).

[159] Jessica R Cohen and Mark D'Esposito, "The segregation and integration of distinct brain networks and their relationship to cognition," Journal of Neuroscience 36, 12083-12094 (2016).

[160] Douglass Godwin, Robert L Barry, and René Marois, "Breakdown of the brain's functional network modularity with awareness," Proceedings of the National Academy of Sciences 112, 3799-3804 (2015).

[161] Javier Gonzalez-Castillo and Peter A Bandettini, "Taskbased dynamic functional connectivity: Recent findings 
and open questions," Neuroimage 180, 526-533 (2018).

[162] Richard F Betzel, Lisa Byrge, Farnaz Zamani Esfahlani, and Daniel P Kennedy, "Temporal fluctuations in the brain's modular architecture during movie-watching," NeuroImage , 116687 (2020).

[163] Richard F Betzel, Maxwell A Bertolero, and Danielle S Bassett, "Non-assortative community structure in resting and task-evoked functional brain networks," bioRxiv , 355016 (2018).

[164] Richard F Betzel, "Organizing principles of whole-brain functional connectivity in zebrafish larvae," Network Neuroscience 4, 234-256 (2020).

[165] Kevin Mann, Courtney L Gallen, and Thomas R Clandinin, "Whole-brain calcium imaging reveals an intrinsic functional network in drosophila," Current Biology 27, 2389-2396 (2017).

[166] Matthieu P Vanni, Allen W Chan, Matilde Balbi, Gergely Silasi, and Timothy H Murphy, "Mesoscale mapping of mouse cortex reveals frequency-dependent cycling between distinct macroscale functional modules," Journal of Neuroscience 37, 7513-7533 (2017).

[167] Laura E Suárez, Ross D Markello, Richard F Betzel, and Bratislav Misic, "Linking structure and function in macroscale brain networks," Trends in Cognitive Sciences (2020).

[168] Andrea Avena-Koenigsberger, Bratislav Misic, and Olaf Sporns, "Communication dynamics in complex brain networks," Nature Reviews Neuroscience 19, 17 (2018).

[169] Alex Fornito and Edward T Bullmore, "Reconciling abnormalities of brain network structure and function in schizophrenia," Current opinion in neurobiology 30, 4450 (2015).

[170] Jill X O'Reilly, Paula L Croxson, Saad Jbabdi, Jerome Sallet, MaryAnn P Noonan, Rogier B Mars, Philip GF Browning, Charles RE Wilson, Anna S Mitchell, Karla L Miller, et al., "Causal effect of disconnection lesions on interhemispheric functional connectivity in rhesus monkeys," Proceedings of the National Academy of Sciences 110, 13982-13987 (2013).

[171] Stewart Heitmann, Matthew J Aburn, and Michael Breakspear, "The brain dynamics toolbox for matlab," Neurocomputing 315, 82-88 (2018).

[172] Farras Abdelnour, Henning U Voss, and Ashish Raj, "Network diffusion accurately models the relationship between structural and functional brain connectivity networks," Neuroimage 90, 335-347 (2014).

[173] Farras Abdelnour, Michael Dayan, Orrin Devinsky, Thomas Thesen, and Ashish Raj, "Functional brain connectivity is predictable from anatomic network's laplacian eigen-structure," NeuroImage 172, 728-739 (2018).

[174] Joaquín Goñi, Andrea Avena-Koenigsberger, Nieves Velez de Mendizabal, Martijn $\mathrm{P}$ van den Heuvel, Richard F Betzel, and Olaf Sporns, "Exploring the morphospace of communication efficiency in complex networks," PLoS One 8, e58070 (2013).

[175] Jonathan J Crofts and Desmond J Higham, "A weighted communicability measure applied to complex brain networks," Journal of the Royal Society Interface 6, 411414 (2009).

[176] Ruben Schmidt, Karl JR LaFleur, Marcel A de Reus, Leonard $\mathrm{H}$ van den Berg, and Martijn $\mathrm{P}$ van den Heuvel, "Kuramoto model simulation of neural hubs and dynamic synchrony in the human cerebral connectome," BMC neuroscience 16, 1-13 (2015).

[177] Andrea Avena-Koenigsberger, Bratislav Mišić, Robert XD Hawkins, Alessandra Griffa, Patric Hagmann, Joaquín Goñi, and Olaf Sporns, "Path ensembles and a tradeoff between communication efficiency and resilience in the human connectome," Brain Structure and Function 222, 603-618 (2017).

[178] Jil Meier, X Zhou, Arjan Hillebrand, Prejaas Tewarie, Cornelis J Stam, and Piet Van Mieghem, "The epidemic spreading model and the direction of information flow in brain networks," NeuroImage 152, 639-646 (2017).

[179] Ashish Raj and Fon Powell, "Models of network spread and network degeneration in brain disorders," Biological Psychiatry: Cognitive Neuroscience and Neuroimaging 3, 788-797 (2018).

[180] Bratislav Mišić, Richard F Betzel, Azadeh Nematzadeh, Joaquin Goni, Alessandra Griffa, Patric Hagmann, Alessandro Flammini, Yong-Yeol Ahn, and Olaf Sporns, "Cooperative and competitive spreading dynamics on the human connectome," Neuron 86, 15181529 (2015).

[181] Caio Seguin, Adeel Razi, and Andrew Zalesky, "Inferring neural signalling directionality from undirected structural connectomes," Nature communications 10, 1-13 (2019).

[182] Caio Seguin, Martijn P Van Den Heuvel, and Andrew Zalesky, "Navigation of brain networks," Proceedings of the National Academy of Sciences 115, 6297-6302 (2018).

[183] Antoine Allard and M Ángeles Serrano, "Navigable maps of structural brain networks across species," PLoS computational biology 16, e1007584 (2020).

[184] Enrico Amico, Kausar Abbas, Duy Anh DuongTran, Uttara Tipnis, Meenusree Rajapandian, Evgeny Chumin, Mario Ventresca, Jaroslaw Harezlak, and Joaquín Goñi, "Towards a mathematical theory of communication for the human connectome," arXiv preprint arXiv:1911.02601 (2019).

[185] Andrea Avena-Koenigsberger, Xiaoran Yan, Artemy Kolchinsky, Martijn van den Heuvel, Patric Hagmann, and Olaf Sporns, "A spectrum of routing strategies for brain networks," PLoS computational biology 15, e1006833 (2019).

[186] Ann M Hermundstad, Danielle S Bassett, Kevin S Brown, Elissa M Aminoff, David Clewett, Scott Freeman, Amy Frithsen, Arianne Johnson, Christine M Tipper, Michael B Miller, et al., "Structural foundations of resting-state and task-based functional connectivity in the human brain," Proceedings of the National Academy of Sciences 110, 6169-6174 (2013).

[187] Luca Cocchi, Ian H Harding, Anton Lord, Christos Pantelis, Murat Yucel, and Andrew Zalesky, "Disruption of structure-function coupling in the schizophrenia connectome," NeuroImage: Clinical 4, 779-787 (2014).

[188] Graham L Baum, Zaixu Cui, David R Roalf, Rastko Ciric, Richard F Betzel, Bart Larsen, Matthew Cieslak, Philip A Cook, Cedric H Xia, Tyler M Moore, et al., "Development of structure-function coupling in human brain networks during youth," Proceedings of the National Academy of Sciences 117, 771-778 (2020).

[189] Caio Seguin, Ye Tian, and Andrew Zalesky, "Network communication models improve the behavioral 
and functional predictive utility of the human structural connectome," bioRxiv (2020).

[190] Richard F Betzel, John D Medaglia, Ari E Kahn, Jonathan Soffer, Daniel R Schonhaut, and Danielle S Bassett, "Structural, geometric and genetic factors predict interregional brain connectivity patterns probed by electrocorticography," Nature biomedical engineering 3, 902-916 (2019).

[191] Farnaz Zamani Esfahlani, Maxwell A Bertolero, Danielle S Bassett, and Richard F Betzel, "Spaceindependent community and hub structure of functional brain networks," NeuroImage 211, 116612 (2020).

[192] Klaus H Maier-Hein, Peter F Neher, Jean-Christophe Houde, Marc-Alexandre Côté, Eleftherios Garyfallidis, Jidan Zhong, Maxime Chamberland, Fang-Cheng Yeh, Ying-Chia Lin, Qing Ji, et al., "The challenge of mapping the human connectome based on diffusion tractography," Nature communications 8, 1-13 (2017).

[193] Colin Reveley, Anil K Seth, Carlo Pierpaoli, Afonso C Silva, David Yu, Richard C Saunders, David A Leopold, and Q Ye Frank, "Superficial white matter fiber systems impede detection of long-range cortical connections in diffusion mr tractography," Proceedings of the National Academy of Sciences 112, E2820-E2828 (2015).

[194] Jonathan D Power, Kelly A Barnes, Abraham Z Snyder, Bradley L Schlaggar, and Steven E Petersen, "Spurious but systematic correlations in functional connectivity mri networks arise from subject motion," Neuroimage 59, 2142-2154 (2012).

[195] Gustavo Deco, Viktor K Jirsa, and Anthony R McIntosh, "Emerging concepts for the dynamical organization of resting-state activity in the brain," Nature Reviews Neuroscience 12, 43-56 (2011).

[196] R Matthew Hutchison, Thilo Womelsdorf, Elena A Allen, Peter A Bandettini, Vince D Calhoun, Maurizio Corbetta, Stefania Della Penna, Jeff H Duyn, Gary H Glover, Javier Gonzalez-Castillo, et al., "Dynamic functional connectivity: promise, issues, and interpretations," Neuroimage 80, 360-378 (2013).

[197] Ricardo Pio Monti, Peter Hellyer, David Sharp, Robert Leech, Christoforos Anagnostopoulos, and Giovanni Montana, "Estimating time-varying brain connectivity networks from functional mri time series," NeuroImage 103, 427-443 (2014).

[198] Catie Chang and Gary H Glover, "Time-frequency dynamics of resting-state brain connectivity measured with fmri," Neuroimage 50, 81-98 (2010).

[199] Xiao Liu and Jeff H Duyn, "Time-varying functional network information extracted from brief instances of spontaneous brain activity," Proceedings of the National Academy of Sciences 110, 4392-4397 (2013).

[200] Martin A Lindquist, Yuting Xu, Mary Beth Nebel, and Brain S Caffo, "Evaluating dynamic bivariate correlations in resting-state fmri: a comparison study and a new approach," NeuroImage 101, 531-546 (2014).

[201] Rikkert Hindriks, Mohit H Adhikari, Yusuke Murayama, Marco Ganzetti, Dante Mantini, Nikos K Logothetis, and Gustavo Deco, "Can sliding-window correlations reveal dynamic functional connectivity in restingstate fmri?" Neuroimage 127, 242-256 (2016).

[202] Morten L Kringelbach and Gustavo Deco, "Brain states and transitions: Insights from computational neuroscience," Cell Reports 32, 108128 (2020).
[203] Vince D Calhoun, Robyn Miller, Godfrey Pearlson, and Tulay Adall, "The chronnectome: time-varying connectivity networks as the next frontier in fmri data discovery," Neuron 84, 262-274 (2014).

[204] Christina B Young, Gal Raz, Daphne Everaerd, Christian F Beckmann, Indira Tendolkar, Talma Hendler, Guillén Fernández, and Erno J Hermans, "Dynamic shifts in large-scale brain network balance as a function of arousal," Journal of Neuroscience 37, 281-290 (2017).

[205] Elena A Allen, Eswar Damaraju, Sergey M Plis, Erik B Erhardt, Tom Eichele, and Vince D Calhoun, "Tracking whole-brain connectivity dynamics in the resting state," Cerebral cortex 24, 663-676 (2014).

[206] Angus Ho Ching Fong, Kwangsun Yoo, Monica D Rosenberg, Sheng Zhang, Chiang-Shan R Li, Dustin Scheinost, $\mathrm{R}$ Todd Constable, and Marvin M Chun, "Dynamic functional connectivity during task performance and rest predicts individual differences in attention across studies," NeuroImage 188, 14-25 (2019).

[207] R Matthew Hutchison, Thilo Womelsdorf, Joseph S Gati, Stefan Everling, and Ravi S Menon, "Restingstate networks show dynamic functional connectivity in awake humans and anesthetized macaques," Human brain mapping 34, 2154-2177 (2013).

[208] Aaron Kucyi and Karen D Davis, "Dynamic functional connectivity of the default mode network tracks daydreaming," Neuroimage 100, 471-480 (2014).

[209] Pablo Barttfeld, Lynn Uhrig, Jacobo D Sitt, Mariano Sigman, Béchir Jarraya, and Stanislas Dehaene, "Signature of consciousness in the dynamics of resting-state brain activity," Proceedings of the National Academy of Sciences 112, 887-892 (2015).

[210] Eswar Damaraju, Elena A Allen, Aysenil Belger, Judith M Ford, S McEwen, DH Mathalon, BA Mueller, GD Pearlson, SG Potkin, A Preda, et al., "Dynamic functional connectivity analysis reveals transient states of dysconnectivity in schizophrenia," NeuroImage: Clinical 5, 298-308 (2014).

[211] Mikko Kivelä, Alex Arenas, Marc Barthelemy, James P Gleeson, Yamir Moreno, and Mason A Porter, "Multilayer networks," Journal of complex networks 2, 203271 (2014).

[212] Danielle S Bassett, Mason A Porter, Nicholas F Wymbs, Scott T Grafton, Jean M Carlson, and Peter J Mucha, "Robust detection of dynamic community structure in networks," Chaos: An Interdisciplinary Journal of Nonlinear Science 23, 013142 (2013).

[213] Marcelo G Mattar, Michael W Cole, Sharon L Thompson-Schill, and Danielle S Bassett, "A functional cartography of cognitive systems," PLoS computational biology 11, e1004533 (2015).

[214] Raphael T Gerraty, Juliet Y Davidow, Karin Foerde, Adriana Galvan, Danielle S Bassett, and Daphna Shohamy, "Dynamic flexibility in striatal-cortical circuits supports reinforcement learning," Journal of Neuroscience 38, 2442-2453 (2018).

[215] Qawi K Telesford, Mary-Ellen Lynall, Jean Vettel, Michael B Miller, Scott T Grafton, and Danielle S Bassett, "Detection of functional brain network reconfiguration during task-driven cognitive states," NeuroImage 142, 198-210 (2016).

[216] Urs Braun, Axel Schäfer, Henrik Walter, Susanne Erk, Nina Romanczuk-Seiferth, Leila Haddad, Janina I Schweiger, Oliver Grimm, Andreas Heinz, Heike Tost, 
et al., "Dynamic reconfiguration of frontal brain networks during executive cognition in humans," Proceedings of the National Academy of Sciences 112, 1167811683 (2015).

[217] Richard F Betzel, Theodore D Satterthwaite, Joshua I Gold, and Danielle S Bassett, "Positive affect, surprise, and fatigue are correlates of network flexibility," Scientific Reports 7, 1-10 (2017).

[218] Karolina Finc, Kamil Bonna, Xiaosong He, David M Lydon-Staley, Simone Kühn, Włodzisław Duch, and Danielle S Bassett, "Dynamic reconfiguration of functional brain networks during working memory training," Nature communications 11, 1-15 (2020).

[219] Urs Braun, Axel Schäfer, Danielle S Bassett, Franziska Rausch, Janina I Schweiger, Edda Bilek, Susanne Erk, Nina Romanczuk-Seiferth, Oliver Grimm, Lena S Geiger, et al., "Dynamic brain network reconfiguration as a potential schizophrenia genetic risk mechanism modulated by nmda receptor function," Proceedings of the National Academy of Sciences 113, 12568-12573 (2016).

[220] Andrew Zalesky, Alex Fornito, Luca Cocchi, Leonardo L Gollo, and Michael Breakspear, "Time-resolved restingstate brain networks," Proceedings of the National Academy of Sciences 111, 10341-10346 (2014).

[221] Zitong Zhang, Qawi K Telesford, Chad Giusti, Kelvin O Lim, and Danielle S Bassett, "Choosing wavelet methods, filters, and lengths for functional brain network construction," PloS one 11, e0157243 (2016).

[222] Andrew Zalesky and Michael Breakspear, "Towards a statistical test for functional connectivity dynamics," Neuroimage 114, 466-470 (2015).

[223] Nora Leonardi and Dimitri Van De Ville, "On spurious and real fluctuations of dynamic functional connectivity during rest," Neuroimage 104, 430-436 (2015).

[224] Timothy O Laumann, Abraham Z Snyder, Anish Mitra, Evan M Gordon, Caterina Gratton, Babatunde Adeyemo, Adrian W Gilmore, Steven M Nelson, Jeff J Berg, Deanna J Greene, et al., "On the stability of bold fmri correlations," Cerebral cortex 27, 4719-4732 (2017).

[225] Raphael Liegeois, Timothy O Laumann, Abraham Z Snyder, Juan Zhou, and BT Thomas Yeo, "Interpreting temporal fluctuations in resting-state functional connectivity mri," Neuroimage 163, 437-455 (2017).

[226] Daniel J Lurie, Daniel Kessler, Danielle S Bassett, Richard F Betzel, Michael Breakspear, Shella Kheilholz, Aaron Kucyi, Raphaël Liégeois, Martin A Lindquist, Anthony Randal McIntosh, et al., "Questions and controversies in the study of time-varying functional connectivity in resting fmri," Network Neuroscience 4, 3069 (2020).

[227] Richard F Betzel and Danielle S Bassett, "Generative models for network neuroscience: prospects and promise," Journal of The Royal Society Interface 14, 20170623 (2017).

[228] Albert-László Barabási and Réka Albert, "Emergence of scaling in random networks," science 286, 509-512 (1999).

[229] Mikail Rubinov, "Constraints and spandrels of interareal connectomes," Nature communications 7, 1-11 (2016).

[230] Vincenzo Nicosia, Petra E Vértes, William R Schafer, Vito Latora, and Edward T Bullmore, "Phase transi- tion in the economically modeled growth of a cellular nervous system," Proceedings of the National Academy of Sciences 110, 7880-7885 (2013).

[231] Petra E Vértes, Aaron F Alexander-Bloch, Nitin Gogtay, Jay N Giedd, Judith L Rapoport, and Edward T Bullmore, "Simple models of human brain functional networks," Proceedings of the National Academy of Sciences 109, 5868-5873 (2012).

[232] Richard F Betzel, Andrea Avena-Koenigsberger, Joaquín Goñi, Ye He, Marcel A De Reus, Alessandra Griffa, Petra E Vértes, Bratislav Mišic, Jean-Philippe Thiran, Patric Hagmann, et al., "Generative models of the human connectome," Neuroimage 124, 1054-1064 (2016).

[233] Mária Ercsey-Ravasz, Nikola T Markov, Camille Lamy, David C Van Essen, Kenneth Knoblauch, Zoltán Toroczkai, and Henry Kennedy, "A predictive network model of cerebral cortical connectivity based on a distance rule," Neuron 80, 184-197 (2013).

[234] Szabolcs Horvát, Răzvan Gămănuț, Mária ErcseyRavasz, Loïc Magrou, Bianca Gămănuț, David C Van Essen, Andreas Burkhalter, Kenneth Knoblauch, Zoltán Toroczkai, and Henry Kennedy, "Spatial embedding and wiring cost constrain the functional layout of the cortical network of rodents and primates," PLoS biology 14, e1002512 (2016).

[235] Jennifer Stiso and Danielle S Bassett, "Spatial embedding imposes constraints on neuronal network architectures," Trends in cognitive sciences 22, 1127-1142 (2018).

[236] Richard F Betzel and Danielle S Bassett, "Specificity and robustness of long-distance connections in weighted, interareal connectomes," Proceedings of the National Academy of Sciences 115, E4880-E4889 (2018).

[237] Aurina Arnatkeviciute, Ben D Fulcher, Stuart Oldham, Jeggan Tiego, Casey Paquola, Zachary F Gerring, Kevin M Aquino, Ziarih Hawi, Beth Johnson, Gareth M Ball, et al., "Genetic influences on hub connectivity of the human connectome," BioRxiv (2020).

[238] Xiaolong Zhang, Urs Braun, Anais Harneit, Zhenxiang Zang, Lena S Geiger, Richard F Betzel, Junfang Chen, Janina Schweiger, Kristina Schwarz, Jonathan Rochus Reinwald, et al., "Generative network models identify biological mechanisms of altered structural brain connectivity in schizophrenia," bioRxiv , 604322 (2019).

[239] Danyal Akarca, Petra E Vértes, Edward T Bullmore, Duncan E Astle, et al., "A generative network model of neurodevelopment," bioRxiv (2020).

[240] Yang-Yu Liu, Jean-Jacques Slotine, and Albert-László Barabási, "Controllability of complex networks," nature 473, 167-173 (2011).

[241] Sean P Cornelius, William L Kath, and Adilson E Motter, "Realistic control of network dynamics," Nature communications 4, 1-9 (2013).

[242] Yang-Yu Liu and Albert-László Barabási, "Control principles of complex systems," Reviews of Modern Physics 88, 035006 (2016).

[243] Fabio Pasqualetti, Sandro Zampieri, and Francesco Bullo, "Controllability metrics, limitations and algorithms for complex networks," IEEE Transactions on Control of Network Systems 1, 40-52 (2014).

[244] Shi Gu, Fabio Pasqualetti, Matthew Cieslak, Qawi K Telesford, B Yu Alfred, Ari E Kahn, John D Medaglia, Jean M Vettel, Michael B Miller, Scott T Grafton, et al., 
"Controllability of structural brain networks," Nature communications 6, 1-10 (2015).

[245] Teresa M Karrer, Jason Z Kim, Jennifer Stiso, Ari E Kahn, Fabio Pasqualetti, Ute Habel, and Danielle S Bassett, "A practical guide to methodological considerations in the controllability of structural brain networks," Journal of Neural Engineering 17, 026031 (2020).

[246] Eli J Cornblath, Evelyn Tang, Graham L Baum, Tyler M Moore, Azeez Adebimpe, David R Roalf, Ruben C Gur, Raquel E Gur, Fabio Pasqualetti, Theodore D Satterthwaite, et al., "Sex differences in network controllability as a predictor of executive function in youth," NeuroImage 188, 122-134 (2019).

[247] John D Medaglia, Denise Y Harvey, Nicole White, Apoorva Kelkar, Jared Zimmerman, Danielle S Bassett, and Roy H Hamilton, "Network controllability in the inferior frontal gyrus relates to controlled language variability and susceptibility to tms," Journal of Neuroscience 38, 6399-6410 (2018).

[248] Lysianne Beynel, Lifu Deng, Courtney A Crowell, Moritz Dannhauer, Hannah Palmer, Susan Hilbig, Angel V Peterchev, Bruce Luber, Sarah H Lisanby, Roberto Cabeza, et al., "Structural controllability predicts functional patterns and brain stimulation benefits associated with working memory," Journal of Neuroscience 40, 6770-6778 (2020).

[249] Evelyn Tang, Chad Giusti, Graham L Baum, Shi Gu, Eli Pollock, Ari E Kahn, David R Roalf, Tyler M Moore, Kosha Ruparel, Ruben C Gur, et al., "Developmental increases in white matter network controllability support a growing diversity of brain dynamics," Nature communications 8, 1-16 (2017).

[250] Won Hee Lee, Amanda Rodrigue, David C Glahn, Danielle S Bassett, and Sophia Frangou, "Heritability and cognitive relevance of structural brain controllability," Cerebral Cortex 30, 3044-3054 (2020).

[251] Jayson Jeganathan, Alistair Perry, Danielle S Bassett, Gloria Roberts, Philip B Mitchell, and Michael Breakspear, "Fronto-limbic dysconnectivity leads to impaired brain network controllability in young people with bipolar disorder and those at high genetic risk," NeuroImage: Clinical 19, 71-81 (2018).

[252] Yoed N Kenett, John D Medaglia, Roger E Beaty, Qunlin Chen, Richard F Betzel, Sharon L Thompson-Schill, and Jiang Qiu, "Driving the brain towards creativity and intelligence: A network control theory analysis," Neuropsychologia 118, 79-90 (2018).

[253] Jason Z Kim, Jonathan M Soffer, Ari E Kahn, Jean M Vettel, Fabio Pasqualetti, and Danielle S Bassett, "Role of graph architecture in controlling dynamical networks with applications to neural systems," Nature physics 14, 91-98 (2018).

[254] Shi Gu, Richard F Betzel, Marcelo G Mattar, Matthew Cieslak, Philip R Delio, Scott $\mathrm{T}$ Grafton, Fabio Pasqualetti, and Danielle S Bassett, "Optimal trajectories of brain state transitions," Neuroimage 148, 305317 (2017).

[255] Richard F Betzel, Shi Gu, John D Medaglia, Fabio Pasqualetti, and Danielle S Bassett, "Optimally controlling the human connectome: the role of network topology," Scientific reports 6, 30770 (2016).

[256] Gang Yan, Petra E Vértes, Emma K Towlson, Yee Lian Chew, Denise S Walker, William R Schafer, and AlbertLászló Barabási, "Network control principles predict neuron function in the caenorhabditis elegans connectome," Nature 550, 519-523 (2017).

[257] Eli J Cornblath, Arian Ashourvan, Jason Z Kim, Richard F Betzel, Rastko Ciric, Azeez Adebimpe, Graham L Baum, Xiaosong He, Kosha Ruparel, Tyler M Moore, et al., "Temporal sequences of brain activity at rest are constrained by white matter structure and modulated by cognitive demands," Communications biology 3, 1-12 (2020).

[258] Urs Braun, Anais Harneit, Giulio Pergola, Tommaso Menara, Axel Schaefer, Richard F Betzel, Zhenxiang Zang, Janina I Schweiger, Kristina Schwarz, Junfang Chen, et al., "Brain state stability during working memory is explained by network control theory, modulated by dopamine $\mathrm{d} 1 / \mathrm{d} 2$ receptor function, and diminished in schizophrenia," arXiv preprint arXiv:1906.09290 (2019).

[259] John D Medaglia, Perry Zurn, Walter SinnottArmstrong, and Danielle S Bassett, "Mind control as a guide for the mind," Nature Human Behaviour 1, 1-8 (2017).

[260] Andrew J Whalen, Sean N Brennan, Timothy D Sauer, and Steven J Schiff, "Observability and controllability of nonlinear networks: The role of symmetry," Physical Review X 5, 011005 (2015).

[261] Junjie Jiang and Ying-Cheng Lai, "Irrelevance of linear controllability to nonlinear dynamical networks," Nature communications 10, 1-10 (2019).

[262] Chengyi Tu, Rodrigo P Rocha, Maurizio Corbetta, Sandro Zampieri, Marco Zorzi, and Samir Suweis, "Warnings and caveats in brain controllability," NeuroImage 176, 83-91 (2018).

[263] Samir Suweis, Chengyi Tu, Rodrigo P Rocha, Sandro Zampieri, Marzo Zorzi, and Maurizio Corbetta, "Brain controllability: Not a slam dunk yet," NeuroImage 200, 552-555 (2019).

[264] TS Evans and Renaud Lambiotte, "Line graphs, link partitions, and overlapping communities," Physical Review E 80, 016105 (2009).

[265] Lucy LW Owen, Tom Hao Chang, and Jeremy R Manning, "High-level cognition during story listening is reflected in high-order dynamic correlations in neural activity patterns," bioRxiv , 763821 (2019).

[266] Marcel A de Reus, Victor M Saenger, René S Kahn, and Martijn $\mathrm{P}$ van den Heuvel, "An edge-centric perspective on the human connectome: link communities in the brain," Philosophical Transactions of the Royal Society B: Biological Sciences 369, 20130527 (2014).

[267] Joshua Faskowitz, Farnaz Zamani Esfahlani, Youngheun Jo, Olaf Sporns, and Richard F Betzel, "Edge-centric functional network representations of human cerebral cortex reveal overlapping system-level architecture," Nature neuroscience (2020).

[268] Farnaz Zamani Esfahlani, Youngheun Jo, Joshua Faskowitz, Lisa Byrge, Daniel Kennedy, Olaf Sporns, and Richard Betzel, "High-amplitude co-fluctuations in cortical activity drive functional connectivity," Proceedings of the National Academy of Sciences (2020).

[269] Youngheun Jo, Farnaz Zamani Esfahlani, Joshua Faskowitz, Evgeny Chumin, Olaf Sporns, and Richard Betzel, "The diversity and multiplexity of edge communities within and between brain systems," bioRxiv (2020). 
[270] Youngheun Jo, Joshua Faskowitz, Farnaz Zamani Esfahlani, Olaf Sporns, and Richard F Betzel, "Subject identification using edge-centric functional connectivity," bioRxiv (2020).

[271] Olaf Sporns, Joshua Faskowitz, Sofia Teixera, and Richard Betzel, "Dynamic expression of brain functional systems disclosed by fine-scale analysis of edge time series," bioRxiv (2020).

[272] Mahshid Najafi, Brenton W McMenamin, Jonathan Z Simon, and Luiz Pessoa, "Overlapping communities reveal rich structure in large-scale brain networks during rest and task conditions," Neuroimage 135, 92-106 (2016).

[273] BT Thomas Yeo, Fenna M Krienen, Michael WL Chee, and Randy L Buckner, "Estimates of segregation and overlap of functional connectivity networks in the human cerebral cortex," Neuroimage 88, 212-227 (2014).

[274] Carsen Stringer, Marius Pachitariu, Nicholas Steinmetz, Charu Bai Reddy, Matteo Carandini, and Kenneth D Harris, "Spontaneous behaviors drive multidimensional, brainwide activity," Science $\mathbf{3 6 4}$ (2019).

[275] William E Allen, Michael Z Chen, Nandini Pichamoorthy, Rebecca H Tien, Marius Pachitariu, Liqun Luo, and Karl Deisseroth, "Thirst regulates motivated behavior through modulation of brainwide neural population dynamics," Science 364 (2019). 